\begin{tabular}{|c|l|}
\hline Title & Lightcone dual ities for curves in the 3-sphere \\
\hline Author(s) & Izumiya, Shyuichi; Jiang, Y ang; Sato, Takami \\
\hline Citation & Hokkaido University Preprint Series in Mathematics, 1007, 1-19 \\
\hline Issue Date & 2012-4-20 \\
\hline DOI & 10.14943/84153 \\
\hline Doc URL & http://hdl.handle.net/2115/69812 \\
\hline Type & bulletin (article) \\
\hline File Information & pre1007.pdf \\
\hline
\end{tabular}

Instructions for use 


\title{
Lightcone dualities for curves in the 3-sphere
}

\author{
Shyuichi Izumiya, Yang Jiang* and Takami Sato
}

April 18, 2012

SHYUICHI IZUMIYA

A. School of Mathematics and Statistics

Northeast Normal University

ChangChun 130024

P.R.CHINA

B. Department of Mathematics

HOKKAIDO UNIVERSITY

SAPPORO 060-0810

JAPAN(THE PRESENT WORK PLACE)

E-mail address: izumiya@math.sci.hokudai.ac.jp

YANG JIANG

A. School of Mathematics and Statistics

Northeast Normal University

ChangChun 130024

P.R.CHINA

B. Department of Mathematics

HOKKAIDO UNIVERSITY

SAPPORO 060-0810

JAPAN

E-mail address: xjiangyang@126.com

TAKAMI SATO

DEPARTMENT OF MATHEMATICS

HOKKAIDO UNIVERSITY

SAPPORO 060-0810

JAPAN

E-mail address: taka_mi@ec.hokudai.ac.jp 


\begin{abstract}
In this paper we consider the curves in the unit 3-sphere. The unit 3-sphere can be canonically embedded in the lightcone and de Sitter 4-space in Loretnz-Minkowski 5space. We investigate these curves in the framework of the theory of Legendrian dualities between pseudo-spheres in Lorentz-Minkowski 5-space.
\end{abstract}

\title{
1 Introduction
}

It has been investigated the evolutes of curves in the unit 2-sphere in [7] from the view point of the Legendrian duality $[4,5]$. It is known that the evolute of a curve in the unit 2-sphere is the dual of the tangent indicatrix of the original curve [11]. The dual curve in the unit 2-sphere is defined to be equidistant by $\pi / 2$ from the original one. For a curve in the unit 3-sphere, however, the dual is a surface. Therefore, the dual of the tangent indicatrix of a curve is a surface which is called the focal surface (or, the focal set) of the original curve. The critical locus of the focal surface is the evolute of the original curve (cf., [11]). We remark that the focal set of a curve in the unit 2-sphere is a curve which is equal to the evolute.

On the other hand, the first author introduced the mandala of Legendrian dualities between pseudo-spheres in Minkowski space $[4,5]$. There are three kinds of pseudo-spheres in LorentzMinkowski 5-space(i.e., the hyperbolic space, the de Sitter space and the lightcone). Especially, if we investigate spacelike submanifolds in the lightcone, those Legendrian dualities are essentially useful (see, also [9]). For the de Sitter space and the lightcone in Lorentz-Minkowski 5 -space, there exist naturally embedded unit 3-spheres. In this paper we investigate the curves in the unit 3-sphere in the framework of the theory of Legendrian dualities between pseudospheres in Lorentz-Minkowski 5-space $([1,2,6,5,10,11,12,13]$, etc.). If we have a regular curve in the unit 3-sphere, then we have the regular curve in the embedded unit 3-sphere in the lightcone or de Sitter space. Therefore, we naturally have the dual hypersurfaces in the lightcone as an application of the duality theorem in [5]. There are two kinds of lightcone dual hypersurfaces of a curve in the unit 3-sphere. The critical value sets of these two hypersurfaces are called the lightcone focal surfaces respectively. The projections of these focal surfaces to unit 3-sphere are different surfaces. In [7] we have shown that the projection images of the critical value sets of lightcone dual surfaces for a curve in the unit 2-sphere coincide with the evolute of the original curve. Therefore, the situation of curves in the unit 3-sphere is quite different from that of curves in the unit 2-sphere. However, the projections of the critical sets of lightcone focal surfaces are equal to the evolute of the curve. In order to clarify such situation, we introduce the notion of discriminant set of higher order for unfoldings of functions of one-variable (see, Section 6).

A brief description of the organization for this paper is as follows: In Section 2, we give basic concepts in this paper. In Section 3, we formulate the Frenet-Serret type formulae for the curves in the unit 3-sphere. We also give the definition of the spherical focal surfaces of the curve in the unit 3-sphere. The spherical evolutes of the curve in the unit 3-sphere is given by the critical sets of the spherical focal surfaces. In Section 4, we define the lightcone dual

* Corresponding author:Jiang Yang, Northeast Normal University; E-mail address:xjiangyang@126.com 2000 Mathematics Subject classification:

Key Words and Phrases: Lorentz-Minkowski 5-space, Lightcone dulaity, The 3-sphere, Singularity 
hypersurfaces and the ligtcone focal surfaces of the curves in the unit 3-sphere. In Section 5, we calculate the conditions for the $A_{k}(k=1,2,3,4)$ singularities for the lightcone height functions of the curves in the unit 3-sphere. In Section 6 , we show that the projections of the critical sets of focal surfaces to the unit 3-sphere are the same, and they are equal to the spherical evolutes of the curve in the unit 3-sphere (Theorem 6.2). We also study the singularities of the lightcone dual surfaces, the lightcone focal surfaces and the spherical evolutes of the curves in the unit 3-sphere which is one of the main results in this paper (cf., Theorem 6.9).

All maps considered here are of class $C^{\infty}$ unless otherwise stated.

\section{Basic concepts}

In this section we give the basic concepts in this paper. Let $\mathbb{R}^{5}$ be a five-dimensional vector space. For any two vectors $\boldsymbol{x}=\left(x_{0}, x_{1}, x_{2}, x_{3}, x_{4}\right), \boldsymbol{y}=\left(y_{0}, y_{1}, y_{2}, y_{3}, y_{4}\right)$ in $\mathbb{R}^{5}$, their pseudo scalar product is defined by $\langle\boldsymbol{x}, \boldsymbol{y}\rangle=-x_{0} y_{0}+x_{1} y_{1}+x_{2} y_{2}+x_{3} y_{3}+x_{4} y_{4}$. The pair $\left(\mathbb{R}^{5},\langle\rangle,\right)$ is called Lorentz-Minkowski 5-space. We denote it as $\mathbb{R}_{1}^{5}$.

For any four vectors $\boldsymbol{x}=\left(x_{0}, x_{1}, x_{2}, x_{3}, x_{4}\right), \boldsymbol{y}=\left(y_{0}, y_{1}, y_{2}, y_{3}, y_{4}\right), \boldsymbol{z}=\left(z_{0}, z_{1}, z_{2}, z_{3}, z_{4}\right), \boldsymbol{w}=$ $\left(w_{0}, w_{1}, w_{2}, w_{3}, w_{4}\right) \in \mathbb{R}_{1}^{5}$, their pseudo vector product is defined by

$$
\boldsymbol{x} \wedge \boldsymbol{y} \wedge \boldsymbol{z} \wedge \boldsymbol{w}=\left|\begin{array}{ccccc}
-\boldsymbol{e}_{0} & \boldsymbol{e}_{1} & \boldsymbol{e}_{2} & \boldsymbol{e}_{3} & \boldsymbol{e}_{4} \\
x_{0} & x_{1} & x_{2} & x_{3} & x_{4} \\
y_{0} & y_{1} & y_{2} & y_{3} & y_{4} \\
z_{0} & z_{1} & z_{2} & z_{3} & z_{4} \\
w_{0} & w_{1} & w_{2} & w_{3} & w_{4}
\end{array}\right|
$$

where $\left\{\boldsymbol{e}_{0}, \boldsymbol{e}_{1}, \boldsymbol{e}_{2}, \boldsymbol{e}_{3}, \boldsymbol{e}_{4}\right\}$ is the canonical basis of $\mathbb{R}_{1}^{5}$. A non-zero vector $\boldsymbol{x} \in \mathbb{R}_{1}^{5}$ is called spacelike, lightlike or timelike if $\langle\boldsymbol{x}, \boldsymbol{x}\rangle>0,\langle\boldsymbol{x}, \boldsymbol{x}\rangle=0$ or $\langle\boldsymbol{x}, \boldsymbol{x}\rangle<0$ respectively. The norm of $\boldsymbol{x} \in \mathbb{R}_{1}^{5}$ is defined by $\|\boldsymbol{x}\|=(\operatorname{sign}(\boldsymbol{x})\langle\boldsymbol{x}, \boldsymbol{x}\rangle)^{1 / 2}$, where $\operatorname{sign}(\boldsymbol{x})$ denotes the signature of $\boldsymbol{x}$ which is given by $\operatorname{sign}(\boldsymbol{x})=1,0$ or -1 when $\boldsymbol{x}$ is a spacelike, lightlike or timelike vector respectively.

Let $\boldsymbol{\gamma}: I \rightarrow \mathbb{R}_{1}^{5}$ be a regular curve in $\mathbb{R}_{1}^{5}$ (i.e., $\dot{\gamma}(t) \neq \mathbf{0}$ for any $t \in I$ ), where $I$ is an open interval. For any $t \in I$, the curve $\gamma$ is called spacelike, lightlike or timelike if $\langle\dot{\gamma}(t), \dot{\gamma}(t)\rangle>$ $0,\langle\dot{\gamma}(t), \dot{\gamma}(t)\rangle=0$ or $\langle\dot{\gamma}(t), \dot{\gamma}(t)\rangle<0$ respectively. We call $\gamma$ a nonlightlike curve if $\gamma$ is a spacelike or timelike curve. The arc-length of a nonlightlike curve $\boldsymbol{\gamma}$ measured from $\gamma\left(t_{0}\right)\left(t_{0} \in I\right)$ is $s(t)=\int_{t_{0}}^{t}\|\dot{\gamma}(t)\| \mathrm{d} t$.

The parameter $s$ is determined such that $\left\|\gamma^{\prime}(s)\right\|=1$ for the nonlightlike curve, where $\gamma^{\prime}(s)=\mathrm{d} \boldsymbol{\gamma} / \mathrm{d} s(s)$ is the unit tangent vector of $\boldsymbol{\gamma}$ at $s$. We define the de Sitter 4-space by

$$
S_{1}^{4}=\left\{\boldsymbol{x} \in \mathbb{R}_{1}^{5} \mid\langle\boldsymbol{x}, \boldsymbol{x}\rangle=1\right\} .
$$

We define the closed lightcone with the vertex $\boldsymbol{a}$ by

$$
L C_{a}=\left\{\boldsymbol{x} \in \mathbb{R}_{1}^{5} \mid\langle\boldsymbol{x}-\boldsymbol{a}, \boldsymbol{x}-\boldsymbol{a}\rangle=0\right\} .
$$

We define the open lightcone at the origin by

$$
L C^{*}=\left\{\boldsymbol{x} \in \mathbb{R}_{1}^{5} \backslash\{\mathbf{0}\} \mid\langle\boldsymbol{x}, \boldsymbol{x}\rangle=0\right\} .
$$

Here we consider the unit sphere in the lightcone defined by

$$
S_{+}^{3}=\left\{\boldsymbol{x} \in L C^{*} \mid x_{0}=1\right\}=\left\{\boldsymbol{x} \in \mathbb{R}_{1}^{5} \mid x_{0}=1, x_{1}^{2}+x_{2}^{2}+x_{3}^{2}+x_{4}^{2}=1\right\},
$$


we call it the lightlike unit sphere. We define the canonical lightcone projection $\pi: L C^{*} \longrightarrow S_{+}^{3}$ by

$$
\pi\left(x_{0}, x_{1}, x_{2}, x_{3}, x_{4}\right)=\left(1, \frac{x_{1}}{x_{0}}, \frac{x_{2}}{x_{0}}, \frac{x_{3}}{x_{0}}, \frac{x_{4}}{x_{0}}\right) .
$$

We also define the 3-dimensional Euclidean unit 3-sphere in $\mathbb{R}_{0}^{4}$ by

$$
S_{0}^{3}=\left\{\boldsymbol{x} \in \mathbb{R}_{1}^{5} \mid x_{0}=0, x_{1}^{2}+x_{2}^{2}+x_{3}^{2}+x_{4}^{2}=1\right\},
$$

where $\mathbb{R}_{0}^{4}=\left\{\boldsymbol{x} \in \mathbb{R}_{1}^{5} \mid x_{0}=0\right\}$ is the Euclidean 4-space.

\section{Curves in the unit 3-sphere and focal surfaces}

Let $\boldsymbol{\gamma}: I \longrightarrow S_{+}^{3}$ be a regular curve. We have a map $\Phi: S_{+}^{3} \rightarrow S_{0}^{3}$ defined by $\Phi(\boldsymbol{v})=\boldsymbol{v}-\boldsymbol{e}_{0}$, which is an isometry. Then we have a regular curve $\bar{\gamma}: I \rightarrow S_{0}^{3}$ defined by $\bar{\gamma}(s)=\Phi(\gamma(s))=$ $\gamma(s)-\boldsymbol{e}_{0}$, so that $\boldsymbol{\gamma}$ and $\bar{\gamma}$ have completely the same geometric properties as spherical curves. Since $\bar{\gamma}$ is a spacelike curve, we can reparameterize it by the arc-length $s$. So we have the unit tangent vector $\boldsymbol{t}(s)=\overline{\boldsymbol{\gamma}}^{\prime}(s)$ of $\overline{\boldsymbol{\gamma}}(s)$. Suppose that $\left\|\boldsymbol{t}^{\prime}(s)\right\| \neq 1$. Then $\left\|\boldsymbol{t}^{\prime}(s)+\overline{\boldsymbol{\gamma}}(s)\right\| \neq 0$, so that we have another unit vector $\boldsymbol{n}(s)=\frac{\boldsymbol{t}^{\prime}(s)+\bar{\gamma}(s)}{\left\|\boldsymbol{t}^{\prime}(s)+\bar{\gamma}(s)\right\|}$. We also define a unit vector by $\boldsymbol{b}(s)=$ $\bar{\gamma}(s) \wedge \boldsymbol{e}_{0} \wedge \boldsymbol{t}(s) \wedge \boldsymbol{n}(s)$, then we have a pseudo-orthonormal frame field $\{\overline{\boldsymbol{\gamma}}(s), \boldsymbol{t}(s), \boldsymbol{n}(s), \boldsymbol{b}(s)\}$ of $\mathbb{R}_{0}^{4}$ along $\bar{\gamma}(s)$. By standard arguments, we have the following Frenet-Serret type formulae.

$$
\left\{\begin{array}{l}
\overline{\boldsymbol{\gamma}}^{\prime}(s)=\boldsymbol{t}(s) \\
\boldsymbol{t}^{\prime}(s)=\kappa_{g}(s) \boldsymbol{n}(s)-\bar{\gamma}(s) \\
\boldsymbol{n}^{\prime}(s)=-\kappa_{g}(s) \boldsymbol{t}(s)+\tau_{g}(s) \boldsymbol{b}(s) \\
\boldsymbol{b}^{\prime}(s)=-\tau_{g}(s) \boldsymbol{n}(s)
\end{array}\right.
$$

where $\kappa_{g}(s)=\left\|\boldsymbol{t}^{\prime}(s)+\bar{\gamma}(s)\right\|$ and $\tau_{g}(s)=-\operatorname{det}\left(\overline{\boldsymbol{\gamma}}(s), \overline{\boldsymbol{\gamma}}^{\prime}(s), \overline{\boldsymbol{\gamma}}^{\prime \prime}(s), \overline{\boldsymbol{\gamma}}^{\prime \prime \prime}(s)\right) / \kappa_{g}^{2}(s)$. We call $\{\overline{\boldsymbol{\gamma}}, \boldsymbol{t}, \boldsymbol{n}, \boldsymbol{b}\}$ a Sabban frame of $\bar{\gamma}$ [8]. Here, $\kappa_{g}$ is called a geodesic curvature and $\tau_{g}$ a geodesic torsion of $\bar{\gamma}$ in $S_{0}^{3}$ respectively.

We now consider the focal surface of a curve $\bar{\gamma}: I \rightarrow S_{0}^{3}$ analogous to the case for curves in Euclidean space. We define $F^{ \pm}: I \times J \rightarrow S_{0}^{3}$ by

$$
F^{ \pm}(s, u)=u \overline{\boldsymbol{\gamma}}(s)+\frac{u}{\kappa_{g}(s)} \boldsymbol{n}(s) \pm \frac{\sqrt{\kappa_{g}^{2}(s)-u^{2}\left(\kappa_{g}^{2}(s)+1\right)}}{\kappa_{g}(s)} \boldsymbol{b}(s) .
$$

We call each image of $F^{ \pm}$the spherical focal surface of $\bar{\gamma}$. We remark that the focal surfaces of $\bar{\gamma}$ satisfies the equations $\left\langle\bar{\gamma}^{\prime}(s), F^{ \pm}(s, u)\right\rangle=\left\langle\bar{\gamma}^{\prime \prime}(s), F^{ \pm}(s, u)\right\rangle=0$. This means that each one of the focal surface $F^{ \pm}(s, u)$ of $\boldsymbol{\gamma}$ is the spherical dual of $\boldsymbol{t}$ in the sense of [10]. By straightforward 
calculations, we have

$$
\begin{aligned}
\frac{\partial F^{ \pm}}{\partial u}(s, u)= & \bar{\gamma}(s)+\frac{1}{\kappa_{g}(s)} \boldsymbol{n}(s) \pm \frac{-u\left(\kappa_{g}^{2}(s)+1\right)}{\kappa_{g}(s) \sqrt{\kappa_{g}^{2}(s)-u^{2}\left(\kappa_{g}^{2}(s)+1\right)}} \boldsymbol{b}(s) \\
\frac{\partial F^{ \pm}}{\partial s}(s, u)= & -\frac{u \kappa_{g}^{\prime}(s) \pm \tau_{g}(s) \kappa_{g}(s) \sqrt{\kappa_{g}^{2}(s)-u^{2}\left(\kappa_{g}^{2}(s)+1\right)}}{\kappa_{g}^{2}(s)} \boldsymbol{n}(s) \\
& +\frac{u \tau_{g}(s) \kappa_{g}(s) \sqrt{\kappa_{g}^{2}(s)-u^{2}\left(\kappa_{g}^{2}(s)+1\right)} \pm u^{2} \kappa_{g}^{\prime}(s)}{\kappa_{g}^{2}(s) \sqrt{\kappa_{g}^{2}(s)-u^{2}\left(\kappa_{g}^{2}(s)+1\right)}} \boldsymbol{b}(s) .
\end{aligned}
$$

It follows that $\left\{\partial F^{ \pm} / \partial u, \partial F^{ \pm} / \partial s\right\}$ is linearly dependent if and only if

$$
\tau_{g}(s) \kappa_{g}(s) \sqrt{\kappa_{g}^{2}(s)-u^{2}\left(\kappa_{g}^{2}(s)+1\right)} \pm u \kappa_{g}^{\prime}(s)=0,
$$

so that we have

$$
u=\frac{ \pm \tau_{g}(s) \kappa_{g}^{2}(s)}{\sqrt{\kappa_{g}^{\prime 2}(s)+\kappa_{g}^{4}(s) \tau_{g}^{2}(s)+\kappa_{g}^{2}(s) \tau_{g}^{2}(s)}} .
$$

Therefore each critical value set of $F^{ \pm}$is given by

$$
\boldsymbol{\varepsilon}_{\bar{\gamma}}^{ \pm}(s)=\frac{ \pm \tau_{g}(s) \kappa_{g}^{2}(s)}{\sqrt{\kappa_{g}^{\prime 2}(s)+\kappa_{g}^{4}(s) \tau_{g}^{2}(s)+\kappa_{g}^{2}(s) \tau_{g}^{2}(s)}}\left\{\bar{\gamma}(s)+\frac{1}{\kappa_{g}(s)} \boldsymbol{n}(s)+\left(\frac{1}{\kappa_{g}(s)}\right)^{\prime} \frac{1}{\tau_{g}(s)} \boldsymbol{b}(s)\right\}
$$

We remark that each curve of $\varepsilon_{\bar{\gamma}}^{ \pm}$satisfies the equations

$$
\left\langle\bar{\gamma}^{\prime}(s), \boldsymbol{\varepsilon}_{\bar{\gamma}}^{ \pm}(s)\right\rangle=\left\langle\bar{\gamma}^{\prime \prime}(s), \boldsymbol{\varepsilon}_{\bar{\gamma}}^{ \pm}(s)\right\rangle=\left\langle\bar{\gamma}^{\prime \prime \prime}(s), \boldsymbol{\varepsilon}_{\bar{\gamma}}^{ \pm}(s)\right\rangle=0 .
$$

In [11] Porteous introduced the notion of the evolute of $\bar{\gamma}$ in the unit 3-sphere. He defined it as the curve satisfies the above equations, so that we call each image of $\varepsilon_{\bar{\gamma}}^{ \pm}$the spherical evolute of $\bar{\gamma}$ in the unit 3-sphere. We remark that $\varepsilon_{\bar{\gamma}}^{-}(s)=-\varepsilon_{\bar{\gamma}}^{+}(s)$. For $s=s_{0}$, we fix that $\boldsymbol{v}_{0}^{ \pm}=\varepsilon_{\bar{\gamma}}^{ \pm}\left(s_{0}\right)$ and $\left\langle\overline{\boldsymbol{\gamma}}\left(s_{0}\right), \boldsymbol{\varepsilon}_{\overline{\boldsymbol{\gamma}}}^{ \pm}\left(s_{0}\right)\right\rangle=c^{ \pm}$. Since $\boldsymbol{v}_{0}^{-}=-\boldsymbol{v}_{0}^{+}$and $c^{-}=-c^{+}$, we have a hyperplane

$$
H P\left(\boldsymbol{v}_{0}^{+}, c^{+}\right)=\left\{\boldsymbol{x} \in \mathbb{R}_{0}^{4} \mid\left\langle\boldsymbol{x}, \boldsymbol{v}_{0}^{+}\right\rangle=c^{+}\right\}=\left\{\boldsymbol{x} \in \mathbb{R}_{0}^{4} \mid\left\langle\boldsymbol{x}, \boldsymbol{v}_{0}^{-}\right\rangle=c^{-}\right\}=H P\left(\boldsymbol{v}_{0}^{-}, c^{-}\right),
$$

so that we have a sphere

$$
S^{2}\left(\boldsymbol{v}_{0}^{ \pm}, c^{ \pm}\right)=H P\left(\boldsymbol{v}_{0}^{ \pm}, c^{ \pm}\right) \cap S_{0}^{3} .
$$

We call $S^{2}\left(\boldsymbol{v}_{0}^{ \pm}, c^{ \pm}\right)$an osculating sphere of $\bar{\gamma}$ at $s_{0}$. Therefore the spherical evolutes $\boldsymbol{\varepsilon}_{\bar{\gamma}}^{ \pm}(s)$ are the loci of the centers of osculating spheres of $\bar{\gamma}$ respectively.

Proposition 3.1. There exists a sphere $S^{2}(\boldsymbol{v}, c) \subset S_{0}^{3}$ such that $\bar{\gamma}(I) \subset S^{2}(\boldsymbol{v}, c)$ if and only if both of the spherical evolutes $\varepsilon_{\bar{\gamma}}^{ \pm}$of $\bar{\gamma}$ are constant.

Proof. If one of the spherical evolutes $\varepsilon_{\bar{\gamma}}^{+}$of $\bar{\gamma}$ is constant, we can set that $\varepsilon_{\bar{\gamma}}^{+}(s)=\boldsymbol{v}^{+}$. In this case another spherical evolute $\boldsymbol{\varepsilon}_{\bar{\gamma}}^{-}$is constant too. Then $\left\langle\bar{\gamma}(s), \boldsymbol{v}^{+}\right\rangle^{\prime}=\left\langle\bar{\gamma}^{\prime}(s), \boldsymbol{v}^{+}\right\rangle=$ $\left\langle\boldsymbol{t}(s), \boldsymbol{\varepsilon}_{\bar{\gamma}}^{+}(s)\right\rangle=0$, so we have $\left\langle\bar{\gamma}(s), \boldsymbol{v}^{+}\right\rangle=c^{+}$and $\bar{\gamma}(I) \subset S^{2}\left(\boldsymbol{v}^{+}, c^{+}\right)$. On the contrary, if $\bar{\gamma}(I) \subset S^{2}(\boldsymbol{v}, c)$, then at any point on $\bar{\gamma}$, the osculating spheres is $S^{2}(\boldsymbol{v}, c)$ itself. So the locus of the centers of osculating spheres of $\bar{\gamma}$ is $\boldsymbol{v}$ and $-\boldsymbol{v}$. Therefore, both of the spherical evolutes $\boldsymbol{\varepsilon}_{\bar{\gamma}}^{ \pm}$of $\bar{\gamma}$ are constant. 


\section{Lightcone duals of curves in the unit 3-sphere}

In [5] the first author introduced the Legendrian dualities between pseudo-spheres in Minkowski space which is a basic tool for the study of hypersurfaces in pseudo-spheres in Minkowski space. We define one-forms $\langle d \boldsymbol{v}, \boldsymbol{w}\rangle=-w_{0} d v_{0}+\sum_{i=1}^{4} w_{i} d v_{i},\langle\boldsymbol{v}, d \boldsymbol{w}\rangle=-v_{0} d w_{0}+$ $\sum_{i=1}^{4} v_{i} d w_{i}$ in $\mathbb{R}_{1}^{5} \times \mathbb{R}_{1}^{5}$ and consider the following two double fibrations:

(1)(a) $L C^{*} \times S_{1}^{4} \supset \Delta_{3}=\{(\boldsymbol{v}, \boldsymbol{w}) \mid\langle\boldsymbol{v}, \boldsymbol{w}\rangle=1\}$,

(b) $\pi_{31}: \Delta_{3} \longrightarrow L C^{*}, \pi_{32}: \Delta_{3} \longrightarrow S_{1}^{4}$,

(c) $\theta_{31}=\langle d \boldsymbol{v}, \boldsymbol{w}\rangle\left|\Delta_{3}, \theta_{32}=\langle\boldsymbol{v}, d \boldsymbol{w}\rangle\right| \Delta_{3}$.

(2)(a) $L C^{*} \times L C^{*} \supset \Delta_{4}=\{(\boldsymbol{v}, \boldsymbol{w}) \mid\langle\boldsymbol{v}, \boldsymbol{w}\rangle=-2\}$,

(b) $\pi_{41}: \Delta_{4} \longrightarrow L C^{*}, \pi_{42}: \Delta_{4} \longrightarrow L C^{*}$,

(c) $\theta_{41}=\langle d \boldsymbol{v}, \boldsymbol{w}\rangle\left|\Delta_{4}, \theta_{42}=\langle\boldsymbol{v}, d \boldsymbol{w}\rangle\right| \Delta_{4}$.

Here, $\pi_{i 1}(\boldsymbol{v}, \boldsymbol{w})=\boldsymbol{v}, \pi_{i 2}(\boldsymbol{v}, \boldsymbol{w})=\boldsymbol{w}$. We remark that $\theta_{i 1}^{-1}(0)$ and $\theta_{i 2}^{-1}(0)$ define the same tangent hyperplane field over $\Delta_{i}$ which is denoted by $K_{i}$, (i=3,4). It has been shown in [5] that each $\left(\Delta_{i}, K_{i}\right)(i=3,4)$ is a contact manifold and both of $\pi_{i j}(j=1,2)$ are Legendrian fibrations. Moreover those contact manifolds are contact diffeomorphic to each other. In [5] we have defined four double fibrations $\left(\Delta_{i}, K_{i}\right)(i=1,2,3,4)$ such that these are contact diffeomorphic to each other. Here, we only use $\left(\Delta_{3}, K_{3}\right)$ and $\left(\Delta_{4}, K_{4}\right)$. If we have an isotropic mapping $i: L \rightarrow \Delta_{i}$ (i.e., $i^{*} \theta_{i 1}=0$ ), we say that $\pi_{i 1}(i(L))$ and $\pi_{i 2}(i(L))$ are $\Delta_{i}$-dual to each other $(i=3,4)$. For detailed properties of Legendrian fibrations, see [1].

We now define hypersurfaces in $L C^{*}$ associated with the curves in $S_{+}^{3}$ or $S_{0}^{3}$. Let $\gamma: I \longrightarrow S_{+}^{3}$ be a unit speed curve. We define $\overline{L D}_{\bar{\gamma}}^{ \pm}: I \times \mathbb{R}^{2} \longrightarrow L C^{*}$ by

$$
\overline{L D}_{\bar{\gamma}}^{ \pm}(s, u, v)=\overline{\boldsymbol{\gamma}}(s)+u \boldsymbol{n}(s)+v \boldsymbol{b}(s) \pm \sqrt{u^{2}+v^{2}+1} \boldsymbol{e}_{0} .
$$

We also define $L D_{\gamma}: I \times \mathbb{R}^{2} \longrightarrow L C^{*}$ by

$$
L D_{\boldsymbol{\gamma}}(s, u, v)=\frac{u^{2}+v^{2}-4}{4} \overline{\boldsymbol{\gamma}}(s)+u \boldsymbol{n}(s)+v \boldsymbol{b}(s)+\frac{u^{2}+v^{2}+4}{4} \boldsymbol{e}_{0} .
$$

Then we have the following proposition.

Proposition 4.1. Under the above notation, we have the followings:

(1) $\bar{\gamma}$ and $\overline{L D}_{\bar{\gamma}}^{ \pm}$are $\Delta_{3}$-dual to each other.

(2) $\gamma$ and $L D_{\gamma}$ are $\Delta_{4}$-dual to each other.

Proof. Consider the mapping $\mathscr{L}_{3}(s, u, v)=\left(\overline{L D}_{\bar{\gamma}}^{ \pm}(s, u, v), \bar{\gamma}(s)\right)$. Then we have

$$
\left\langle\overline{L D}_{\bar{\gamma}}^{ \pm}(s, u, v), \bar{\gamma}(s)\right\rangle=\langle\bar{\gamma}(s), \bar{\gamma}(s)\rangle=1
$$

and

$$
\mathscr{L}_{3}^{*} \theta_{32}=\left\langle\overline{L D}_{\bar{\gamma}}^{ \pm}(s, u, v), \bar{\gamma}^{\prime}(s)\right\rangle d s=\left\langle\overline{L D}_{\bar{\gamma}}^{ \pm}(s, u, v), \boldsymbol{t}(s)\right\rangle d s=0 .
$$

The assertion (1) holds.

We also consider the mapping $\mathscr{L}_{4}(s, u, v)=\left(L D_{\boldsymbol{\gamma}}(s, u, v), \boldsymbol{\gamma}(s)\right)$. Since $\left\langle\boldsymbol{\gamma}(s), \boldsymbol{e}_{0}\right\rangle=-1$ and $\langle\gamma(s), \bar{\gamma}(s)\rangle=1$, we have $\left\langle L D_{\gamma}(s, u, v), \gamma(s)\right\rangle=\left(u^{2}+v^{2}\right) / 4-1-\left(\left(u^{2}+v^{2}\right) / 4+1\right)=-2$. Moreover, we have

$$
\left.\mathscr{L}_{4}^{*} \theta_{42}=\left\langle L D_{\gamma}(s, u, v)\right), \boldsymbol{\gamma}^{\prime}(s)\right\rangle d s=\left\langle L D_{\gamma}(s, u, v), \boldsymbol{t}(s)\right\rangle d s=0 .
$$


This completes the proof.

We call each one of $\overline{L D}_{\bar{\gamma}}^{ \pm}$the Lightcone dual haypersurface of the de Sitter spherical curve $\bar{\gamma}$ and $L D_{\gamma}$ the Lightcone dual hypersurface of the lightlike spherical curve $\gamma$. Then we have two mappings $\pi \circ \overline{L D}_{\bar{\gamma}}^{ \pm}: I \times \mathbb{R}^{2} \rightarrow S_{+}^{3}$ and $\pi \circ L D_{\gamma}: I \times \mathbb{R}^{2} \rightarrow S_{+}^{3}$ defined by

$$
\begin{aligned}
\pi \circ \overline{L D}_{\bar{\gamma}}^{ \pm}(s, u, v) & = \pm\left(\frac{1}{\sqrt{u^{2}+v^{2}+1}} \bar{\gamma}(s)+\frac{u}{\sqrt{u^{2}+v^{2}+1}} \boldsymbol{n}(s)+\frac{v}{\sqrt{u^{2}+v^{2}+1}} \boldsymbol{b}(s)\right)+\boldsymbol{e}_{0}, \\
\pi \circ L D_{\gamma}(s, u, v) & =\frac{u^{2}+v^{2}-4}{u^{2}+v^{2}+4} \bar{\gamma}(s)+\frac{4 u}{u^{2}+v^{2}+4} \boldsymbol{n}(s)+\frac{4 v}{u^{2}+v^{2}+4} \boldsymbol{b}(s)+\boldsymbol{e}_{0} .
\end{aligned}
$$

In this paper we consider the singularities of these dual surfaces and mappings. By the Frenet-Serret type formulae, we have

$$
\begin{aligned}
\frac{\partial \overline{L D}_{\bar{\gamma}}^{ \pm}}{\partial u}(s, u, v) & =\boldsymbol{n}(s) \pm \frac{u}{\sqrt{1+u^{2}+v^{2}}} \boldsymbol{e}_{0} \\
\frac{\partial \overline{L D_{\bar{\gamma}}^{ \pm}}}{\partial v}(s, u, v) & =\boldsymbol{b}(s) \pm \frac{v}{\sqrt{1+u^{2}+v^{2}}} \boldsymbol{e}_{0} \\
\frac{\partial \overline{L D_{\bar{\gamma}}^{ \pm}}}{\partial s}(s, u, v) & =\left(1-u \kappa_{g}(s)\right) \boldsymbol{t}(s)-v \tau_{g}(s) \boldsymbol{n}(s)+u \tau_{g}(s) \boldsymbol{b}(s) \\
\frac{\partial L D_{\gamma}}{\partial u}(s, u, v) & =\frac{u}{2} \bar{\gamma}(s)+\boldsymbol{n}(s)+\frac{u}{2} \boldsymbol{e}_{0} \\
\frac{\partial L D_{\gamma}}{\partial v}(s, u, v) & =\frac{v}{2} \bar{\gamma}(s)+\boldsymbol{b}(s)+\frac{v}{2} \boldsymbol{e}_{0} \\
\frac{\partial L D_{\gamma}}{\partial s}(s, u, v) & =\frac{u^{2}+v^{2}-4 u \kappa_{g}(s)-4}{4} \boldsymbol{t}(s)-v \tau_{g}(s) \boldsymbol{n}(s)+u \tau_{g}(s) \boldsymbol{b}(s) .
\end{aligned}
$$

Then we have the following proposition.

Proposition 4.2. Let $\gamma: I \longrightarrow S_{+}^{3}$ be a unit speed curve. Then we have the followings:

(1) $(s, u, v)$ is a singular point of $\overline{L D}_{\bar{\gamma}}^{ \pm}$if and only if $u=1 / \kappa_{g}(s)$.

(2) $(s, u, v)$ is a singular point of $L D_{\gamma}$ if and only if $v= \pm \sqrt{4+4 u \kappa_{g}(s)-u^{2}}$.

Proof. By the above calculations, $\partial \overline{L D}_{\bar{\gamma}}^{ \pm} / \partial u(s, u, v), \partial \overline{L D}_{\bar{\gamma}}^{ \pm} / \partial v(s, u, v)$ and $\partial \overline{L D}_{\bar{\gamma}}^{ \pm} / \partial s(s, u, v)$ are linearly dependent if and only if $u=1 / \kappa_{g}(s)$. The assertion (1) follows. By the similar reason, we have the assertion (2).

Therefore, the critical value sets of the above dual surfaces are given by

$$
\begin{aligned}
C\left(\overline{L D_{\bar{\gamma}}^{ \pm}}\right) & =\left\{\bar{\gamma}(s)+\frac{1}{\kappa_{g}(s)} \boldsymbol{n}(s)+v \boldsymbol{b}(s) \pm \sqrt{\frac{1+\kappa_{g}^{2}(s)+v^{2} \kappa_{g}^{2}(s)}{\kappa_{g}^{2}(s)}} \boldsymbol{e}_{0} \mid v \in \mathbb{R}, s \in I, \kappa_{g}(s) \neq 0\right\}, \\
C\left(L D_{\gamma}\right)^{ \pm} & =\left\{\kappa_{g}(s) u \overline{\boldsymbol{\gamma}}(s)+u \boldsymbol{n}(s) \pm \sqrt{\left.4+4 u \kappa_{g}(s)-u^{2} \boldsymbol{b}(s)+\left(\kappa_{g}(s) u+2\right) \boldsymbol{e}_{0} \mid u \in \mathbb{R}, s \in I\right\} .}\right.
\end{aligned}
$$

We respectively denote that

$$
\begin{aligned}
L F_{\bar{\gamma}}^{ \pm}(s, v) & =\bar{\gamma}(s)+\frac{1}{\kappa_{g}(s)} \boldsymbol{n}(s)+v \boldsymbol{b}(s) \pm \sqrt{\frac{1+\kappa_{g}^{2}(s)+v^{2} \kappa_{g}^{2}(s)}{\kappa_{g}^{2}(s)}} \boldsymbol{e}_{0}, \\
L F_{\gamma}^{ \pm}(s, u) & =\kappa_{g}(s) u \overline{\boldsymbol{\gamma}}(s)+u \boldsymbol{n}(s) \pm \sqrt{4+4 u \kappa_{g}(s)-u^{2}} \boldsymbol{b}(s)+\left(\kappa_{g}(s) u+2\right) \boldsymbol{e}_{0},
\end{aligned}
$$


where we have the relation $v= \pm \sqrt{4+4 u \kappa_{g}(s)-u^{2}}$. We respectively call each one of $L F_{\bar{\gamma}}^{ \pm}$ the lightcone focal surface of the de Sitter spherical curve $\bar{\gamma}$ and each one of $L F_{\gamma}^{ \pm}$the ligtcone focal surface of the lightcone spherical curve $\gamma$. Then the projections of these surfaces to $S_{+}^{3}$ are given as follows:

$$
\begin{aligned}
\pi\left(C\left(\overline{L D}_{\bar{\gamma}}^{ \pm}\right)\right) & =\left\{\frac{ \pm\left(\kappa_{g}(s) \bar{\gamma}(s)+\boldsymbol{n}(s)+v \kappa_{g}(s) \boldsymbol{b}(s)\right)}{\left.\sqrt{1+\kappa_{g}^{2}(s)+v^{2} \kappa_{g}^{2}(s)}+\boldsymbol{e}_{0} \mid v \in \mathbb{R}, s \in I, \kappa_{g}(s) \neq 0\right\},}\right. \\
\pi\left(C\left(L D_{\gamma}\right)^{ \pm}\right) & =\left\{\frac{u \kappa_{g}(s) \bar{\gamma}(s)+u \boldsymbol{n}(s) \pm \sqrt{4+4 u \kappa_{g}(s)-u^{2}} \boldsymbol{b}(s)}{\kappa_{g}(s) u+2}+\boldsymbol{e}_{0} \mid u \in \mathbb{R}, s \in I\right\} .
\end{aligned}
$$

On the other hand, we define $\tilde{\pi}=\Phi \circ \pi: L C^{*} \rightarrow S_{0}^{3}$. By the previous calculations, $\tilde{\pi}\left(C\left(\overline{L D}_{\bar{\gamma}}^{ \pm}\right)\right)$is different from $\tilde{\pi}\left(C\left(L D_{\gamma}\right)^{ \pm}\right)$. In [7], it was shown that the projections of the critical value sets of the lightcone dual surfaces of $\gamma$ and $\bar{\gamma}$ are the same for a curve $\gamma: I \rightarrow S_{+}^{2}$. Moreover, it is equal to the spherical evolute of $\bar{\gamma}$. Therefore, the situation for curves in $S_{+}^{3}$ is quite different from that for curves in $S_{+}^{2}$.

\section{$5 \quad$ Lightcone height functions}

In order to study the singularities of Lightcone dual surfaces of spherical curves, we introduce two families of functions and apply the theory of unfoldings. Let $\boldsymbol{\gamma}: I \longrightarrow S_{+}^{3}$ be a unit speed curve, then we define two families of functions as follows:

$$
\begin{array}{ll}
\bar{H}: I \times L C^{*} \longrightarrow \mathbb{R}, & \bar{H}(s, \boldsymbol{v})=\langle\bar{\gamma}(s), \boldsymbol{v}\rangle-1, \\
H: I \times L C^{*} \longrightarrow \mathbb{R}, & H(s, \boldsymbol{v})=\langle\gamma(s), \boldsymbol{v}\rangle+2 .
\end{array}
$$

We call $\bar{H}$ a lightcone height function of the de Sitter spherical curve $\bar{\gamma}$. For any fixed $\boldsymbol{v} \in L C^{*}$, we denote $\bar{h}_{\boldsymbol{v}}(s)=\bar{H}(s, \boldsymbol{v})$. We call $H$ a lightcone height function of the lightlike spherical curve $\boldsymbol{\gamma}$. For any fixed $\boldsymbol{v} \in L C^{*}$, we denote $h_{\boldsymbol{v}}(s)=H(s, \boldsymbol{v})$. Then we have the following two propositions on $h_{\boldsymbol{v}}$ and $\bar{h}_{\boldsymbol{v}}$.

For simplication, we denote $\rho(s)=\sqrt{\left(\kappa_{g}^{4}(s) \tau_{g}^{2}(s)+\kappa_{g}^{2}(s) \tau_{g}^{2}(s)+\kappa_{g}^{\prime 2}(s)\right) / \kappa_{g}^{4}(s) \tau_{g}^{2}(s)}$ and $\sigma^{ \pm}(s)=\left(\kappa_{g}^{2}(s) \tau_{g}(s) \pm \sqrt{\kappa_{g}^{\prime 2}(s)+\kappa_{g}^{2}(s) \tau_{g}^{2}(s)+\kappa_{g}^{4}(s) \tau_{g}^{2}(s)}\right) /\left(\kappa_{g}^{\prime 2}(s)+\kappa_{g}^{2}(s) \tau_{g}^{2}(s)\right)$.

Proposition 5.1. Let $\gamma: I \longrightarrow S_{+}^{3}$ be a unit speed curve, then we have the followings:

(1) $\bar{h}_{\boldsymbol{v}}(s)=0$ if and only if there exist $\lambda, \mu, \xi, \eta \in \mathbb{R}$ with $\eta^{2}=1+\lambda^{2}+\mu^{2}+\xi^{2}$ such that $\boldsymbol{v}=\overline{\boldsymbol{\gamma}}(s)+\lambda \boldsymbol{t}(s)+\mu \boldsymbol{n}(s)+\xi \boldsymbol{b}(s)+\eta \boldsymbol{e}_{0}$.

(2) $\bar{h}_{\boldsymbol{v}}(s)=\bar{h}_{\boldsymbol{v}}^{\prime}(s)=0$ if and only if there exist $\mu, \xi, \eta \in \mathbb{R}$ with $\eta^{2}=1+\mu^{2}+\xi^{2}$ such that $\boldsymbol{v}=\bar{\gamma}(s)+\mu \boldsymbol{n}(s)+\xi \boldsymbol{b}(s)+\eta \boldsymbol{e}_{0}=\bar{\gamma}(s)+\mu \boldsymbol{n}(s)+\xi \boldsymbol{b}(s) \pm \sqrt{1+\mu^{2}+\xi^{2}} \boldsymbol{e}_{0}$.

(3) $\bar{h}_{\boldsymbol{v}}(s)=\bar{h}_{\boldsymbol{v}}^{\prime}(s)=\bar{h}_{\boldsymbol{v}}^{\prime \prime}(s)=0$ if and only if $\kappa_{g}(s) \neq 0$ and

$$
\boldsymbol{v}=\bar{\gamma}(s)+\frac{1}{\kappa_{g}(s)} \boldsymbol{n}(s)+\xi \boldsymbol{b}(s) \pm \sqrt{\frac{1+\kappa_{g}^{2}(s)+\kappa_{g}^{2}(s) \xi^{2}}{\kappa_{g}^{2}(s)}} \boldsymbol{e}_{0}
$$

(4) $\bar{h}_{\boldsymbol{v}}(s)=\bar{h}_{\boldsymbol{v}}^{\prime}(s)=\bar{h}_{\boldsymbol{v}}^{\prime \prime}(s)=\bar{h}_{\boldsymbol{v}}^{\prime \prime \prime}(s)=0$ if and only if $\kappa_{g}(s) \neq 0, \tau_{g}(s) \neq 0$ and

$$
\boldsymbol{v}=\bar{\gamma}(s)+\frac{1}{\kappa_{g}(s)} \boldsymbol{n}(s)-\frac{\kappa_{g}^{\prime}(s)}{\kappa_{g}^{2}(s) \tau_{g}(s)} \boldsymbol{b}(s) \pm \rho(s) \boldsymbol{e}_{0}
$$


(5) $\bar{h}_{\boldsymbol{v}}(s)=\bar{h}_{\boldsymbol{v}}^{\prime}(s)=\bar{h}_{\boldsymbol{v}}^{\prime \prime}(s)=\bar{h}_{\boldsymbol{v}}^{\prime \prime \prime}(s)=\bar{h}_{\boldsymbol{v}}^{(4)}(s)=0$ if and only if $\kappa_{g}(s) \neq 0, \tau_{g}(s) \neq 0$,

$$
\left(\left(\frac{-1}{\kappa_{g}(s)}\right)^{\prime} \frac{1}{\tau_{g}(s)}\right)^{\prime}-\frac{\tau_{g}(s)}{\kappa_{g}(s)}=0
$$

and

$$
\boldsymbol{v}=\bar{\gamma}(s)+\frac{1}{\kappa_{g}(s)} \boldsymbol{n}(s)-\frac{\kappa_{g}^{\prime}(s)}{\kappa_{g}^{2}(s) \tau_{g}(s)} \boldsymbol{b}(s) \pm \rho(s) \boldsymbol{e}_{0}
$$

(6) $\bar{h}_{\boldsymbol{v}}(s)=\bar{h}_{\boldsymbol{v}}^{\prime}(s)=\bar{h}_{\boldsymbol{v}}^{\prime \prime}(s)=\bar{h}_{\boldsymbol{v}}^{\prime \prime \prime}(s)=\bar{h}_{\boldsymbol{v}}^{(4)}(s)=\bar{h}_{\boldsymbol{v}}^{(5)}(s)=0$ if and only if $\kappa_{g}(s) \neq 0, \tau_{g}(s) \neq 0$,

$$
\left(\left(\frac{-1}{\kappa_{g}(s)}\right)^{\prime} \frac{1}{\tau_{g}(s)}\right)^{\prime}-\frac{\tau_{g}(s)}{\kappa_{g}(s)}=\left\{\left(\left(\frac{-1}{\kappa_{g}(s)}\right)^{\prime} \frac{1}{\tau_{g}(s)}\right)^{\prime}-\frac{\tau_{g}(s)}{\kappa_{g}(s)}\right\}^{\prime}=0
$$

and

$$
\boldsymbol{v}=\overline{\boldsymbol{\gamma}}(s)+\frac{1}{\kappa_{g}(s)} \boldsymbol{n}(s)-\frac{\kappa_{g}^{\prime}(s)}{\kappa_{g}^{2}(s) \tau_{g}(s)} \boldsymbol{b}(s) \pm \rho(s) \boldsymbol{e}_{0}
$$

Proof. (1) Since $\boldsymbol{v} \in L C^{*}$, there exist $\omega, \lambda, \mu, \xi, \eta \in \mathbb{R}$ with $\omega^{2}+\lambda^{2}+\mu^{2}+\xi^{2}-\eta^{2}=0$ such that $\boldsymbol{v}=\omega \bar{\gamma}(s)+\lambda \boldsymbol{t}(s)+\mu \boldsymbol{n}(s)+\xi \boldsymbol{b}(s)+\eta \boldsymbol{e}_{0}$. From $\bar{h}_{\boldsymbol{v}}(s)=\langle\bar{\gamma}(s), \boldsymbol{v}\rangle-1=0$, we have $\omega=1$. So $\boldsymbol{v}=\overline{\boldsymbol{\gamma}}(s)+\lambda \boldsymbol{t}(s)+\boldsymbol{\mu} \boldsymbol{n}(s)+\xi \boldsymbol{b}(s)+\eta \boldsymbol{e}_{0}$ and $\eta^{2}=1+\lambda^{2}+\mu^{2}+\xi^{2}$. The converse direction also holds.

(2) Since $\bar{h}_{\boldsymbol{v}}^{\prime}(s)=\langle\boldsymbol{t}(s), \boldsymbol{v}\rangle, \bar{h}_{\boldsymbol{v}}(s)=\bar{h}_{\boldsymbol{v}}^{\prime}(s)=0$ if and only if

$$
\bar{h}_{\boldsymbol{v}}^{\prime}(s)=\langle\boldsymbol{t}(s), \boldsymbol{v}\rangle=\left\langle\boldsymbol{t}(s), \overline{\boldsymbol{\gamma}}(s)+\lambda \boldsymbol{t}(s)+\mu \boldsymbol{n}(s)+\xi \boldsymbol{b}(s)+\eta \boldsymbol{e}_{0}\right\rangle=\lambda=0 .
$$

It follows from the fact $\eta^{2}=1+\mu^{2}+\xi^{2}$ that $\eta= \pm \sqrt{1+\mu^{2}+\xi^{2}}$. Then we have $\boldsymbol{v}=$ $\bar{\gamma}(s)+\mu \boldsymbol{n}(s)+\xi \boldsymbol{b}(s)+\eta \boldsymbol{e}_{0}=\bar{\gamma}(s)+\mu \boldsymbol{n}(s)+\xi \boldsymbol{b}(s) \pm \sqrt{1+\mu^{2}+\xi^{2}} \boldsymbol{e}_{0}$.

(3) Since $\bar{h}_{\boldsymbol{v}}^{\prime \prime}(s)=\left\langle\kappa_{g}(s) \boldsymbol{n}(s)-\bar{\gamma}(s), \boldsymbol{v}\right\rangle, \bar{h}_{\boldsymbol{v}}(s)=\bar{h}_{\boldsymbol{v}}^{\prime}(s)=\bar{h}_{\boldsymbol{v}}^{\prime \prime}(s)=0$ if and only if

$$
\bar{h}_{\boldsymbol{v}}^{\prime \prime}(s)=\left\langle\kappa_{g}(s) \boldsymbol{n}(s)-\bar{\gamma}(s), \bar{\gamma}(s)+\mu \boldsymbol{n}(s)+\xi \boldsymbol{b}(s) \pm \sqrt{1+\mu^{2}+\xi^{2}} \boldsymbol{e}_{0}\right\rangle=\kappa_{g}(s) \mu-1=0 .
$$

Then we have $\kappa_{g}(s) \neq 0, \mu=1 / \kappa_{g}(s)$ and

$$
\boldsymbol{v}=\bar{\gamma}(s)+\boldsymbol{n}(s) / \kappa_{g}(s)+\xi \boldsymbol{b}(s) \pm \sqrt{\left(1+\kappa_{g}^{2}(s)+\kappa_{g}^{2}(s) \xi^{2}\right) / \kappa_{g}^{2}(s)} \boldsymbol{e}_{0}
$$

(4) Since $\bar{h}_{\boldsymbol{v}}^{\prime \prime \prime}(s)=\left\langle\kappa_{g}^{\prime}(s) \boldsymbol{n}(s)-\left(\kappa_{g}^{2}(s)+1\right) \boldsymbol{t}(s)+\kappa_{g}(s) \tau_{g}(s) \boldsymbol{b}(s), \boldsymbol{v}\right\rangle, \bar{h}_{\boldsymbol{v}}(s)=\bar{h}_{\boldsymbol{v}}^{\prime}(s)=\bar{h}_{\boldsymbol{v}}^{\prime \prime}(s)=$ $\bar{h}_{\boldsymbol{v}}^{\prime \prime \prime}(s)=0$ if and only if

$$
\begin{aligned}
\bar{h}_{\boldsymbol{v}}^{\prime \prime \prime}(s)= & \left\langle\kappa_{g}^{\prime}(s) \boldsymbol{n}(s)-\left(\kappa_{g}^{2}(s)+1\right) \boldsymbol{t}(s)+\kappa_{g}(s) \tau_{g}(s) \boldsymbol{b}(s),\right. \\
& \left.\bar{\gamma}(s)+\boldsymbol{n}(s) / \kappa_{g}(s)+\xi \boldsymbol{b}(s) \pm \sqrt{\left(1+\kappa_{g}^{2}(s)+\kappa_{g}^{2}(s) \xi^{2}\right) / \kappa_{g}^{2}(s)} \boldsymbol{e}_{0}\right\rangle \\
= & \kappa_{g}^{\prime}(s) / \kappa_{g}(s)+\kappa_{g}(s) \tau_{g}(s) \xi=0 .
\end{aligned}
$$

Then we have $\kappa_{g}(s) \neq 0, \tau_{g}(s) \neq 0, \xi=-\kappa_{g}^{\prime}(s) / \kappa_{g}^{2}(s) \tau_{g}(s)$ and $\boldsymbol{v}=\overline{\boldsymbol{\gamma}}(s)+\boldsymbol{n}(s) / \kappa_{g}(s)-$ $\kappa_{g}^{\prime}(s) \boldsymbol{b}(s) / \kappa_{g}^{2}(s) \tau_{g}(s) \pm \rho(s) \boldsymbol{e}_{0}$. 
(5) Since $\bar{h}_{\boldsymbol{v}}^{(4)}(s)=\left\langle\left(\kappa_{g}^{\prime \prime}(s)-\kappa_{g}^{3}(s)-\kappa_{g}(s)-\kappa_{g}(s) \tau_{g}^{2}(s)\right) \boldsymbol{n}(s)-3 \kappa_{g}(s) \kappa_{g}^{\prime}(s) \boldsymbol{t}(s)+\left(2 \kappa_{g}^{\prime}(s) \tau_{g}(s)+\right.\right.$ $\left.\left.\kappa_{g}(s) \tau_{g}^{\prime}(s)\right) \boldsymbol{b}(s)+\left(1+\kappa_{g}^{2}(s)\right) \bar{\gamma}(s), \boldsymbol{v}\right\rangle, \bar{h}_{\boldsymbol{v}}(s)=\bar{h}_{\boldsymbol{v}}^{\prime}(s)=\bar{h}_{\boldsymbol{v}}^{\prime \prime}(s)=\bar{h}_{\boldsymbol{v}}^{\prime \prime \prime}(s)=\bar{h}_{\boldsymbol{v}}^{(4)}(s)=0$ if and only if

$$
\begin{aligned}
\bar{h}_{\boldsymbol{v}}^{(4)}(s)= & \left\langle\left(\kappa_{g}^{\prime \prime}(s)-\kappa_{g}^{3}(s)-\kappa_{g}(s)-\kappa_{g}(s) \tau_{g}^{2}(s)\right) \boldsymbol{n}(s)-3 \kappa_{g}(s) \kappa_{g}^{\prime}(s) \boldsymbol{t}(s)\right. \\
& +\left(2 \kappa_{g}^{\prime}(s) \tau_{g}(s)+\kappa_{g}(s) \tau_{g}^{\prime}(s)\right) \boldsymbol{b}(s)+\left(1+\kappa_{g}^{2}(s)\right) \overline{\boldsymbol{\gamma}}(s) \\
\left.\overline{\boldsymbol{\gamma}}(s)+\frac{1}{\kappa_{g}(s)} \boldsymbol{n}(s)-\frac{\kappa_{g}^{\prime}(s)}{\kappa_{g}^{2}(s) \tau_{g}(s)} \boldsymbol{b}(s) \pm \rho(s) \boldsymbol{e}_{0}\right\rangle & \\
& =\frac{\kappa_{g}(s) \kappa_{g}^{\prime \prime}(s) \tau_{g}(s)-2 \kappa_{g}^{\prime 2}(s) \tau_{g}(s)-\kappa_{g}(s) \kappa_{g}^{\prime}(s) \tau_{g}^{\prime}(s)-\kappa_{g}^{2}(s) \tau_{g}^{3}(s)}{\kappa_{g}^{2}(s) \tau_{g}(s)}=0 .
\end{aligned}
$$

This is equivalent to the condition $\left(\left(-1 / \kappa_{g}(s)\right)^{\prime} / \tau_{g}(s)\right)^{\prime}-\tau_{g}(s) / \kappa_{g}(s)=0$. Then we have $\kappa_{g}(s) \neq 0, \tau_{g}(s) \neq 0,\left(\left(-1 / \kappa_{g}(s)\right)^{\prime} / \tau_{g}(s)\right)^{\prime}-\tau_{g}(s) / \kappa_{g}(s)=0$ and $\boldsymbol{v}=\bar{\gamma}(s)+\boldsymbol{n}(s) / \kappa_{g}(s)-$ $\kappa_{g}^{\prime}(s) \boldsymbol{b}(s) / \kappa_{g}^{2}(s) \tau_{g}(s) \pm \rho(s) \boldsymbol{e}_{0}$.

(6) Since $\bar{h}_{\boldsymbol{v}}^{(5)}(s)=\left\langle\left(\kappa_{g}^{4}(s)+2 \kappa_{g}^{2}(s)+\kappa_{g}^{2}(s) \tau_{g}^{2}(s)+1-3 \kappa_{g}^{\prime 2}(s)-4 \kappa_{g}(s) \kappa_{g}^{\prime \prime}(s)\right) \boldsymbol{t}(s)+\left(\kappa_{g}^{\prime \prime \prime}(s)-\right.\right.$ $\left.\kappa_{g}^{\prime}(s)-6 \kappa_{g}^{2}(s) \kappa_{g}^{\prime}(s)-3 \kappa_{g}^{\prime}(s) \tau_{g}^{2}(s)-3 \kappa_{g}(s) \tau_{g}(s) \tau_{g}^{\prime}(s)\right) \boldsymbol{n}(s)+\left(3 \kappa_{g}^{\prime \prime}(s) \tau_{g}(s)+3 \kappa_{g}^{\prime}(s) \tau_{g}^{\prime}(s)+\kappa_{g}(s) \tau_{g}^{\prime \prime}(s)-\right.$ $\left.\left.\kappa_{g}(s) \tau_{g}(s)-\kappa_{g}^{3}(s) \tau_{g}(s)-\kappa_{g}(s) \tau_{g}^{3}(s)\right) \boldsymbol{b}(s)+5 \kappa_{g}(s) \kappa_{g}^{\prime}(s) \bar{\gamma}(s), \boldsymbol{v}\right\rangle, \bar{h}_{\boldsymbol{v}}(s)=\bar{h}_{\boldsymbol{v}}^{\prime}(s)=\bar{h}_{\boldsymbol{v}}^{\prime \prime}(s)=\bar{h}_{\boldsymbol{v}}^{\prime \prime \prime}(s)=$ $\bar{h}_{\boldsymbol{v}}^{(4)}(s)=\bar{h}_{\boldsymbol{v}}^{(5)}(s)=0$ if and only if

$$
\begin{aligned}
\bar{h}_{\boldsymbol{v}}^{(5)}(s)= & \left\langle\left(\kappa_{g}^{4}(s)+2 \kappa_{g}^{2}(s)+\kappa_{g}^{2}(s) \tau_{g}^{2}(s)+1-3 \kappa_{g}^{\prime 2}(s)-4 \kappa_{g}(s) \kappa_{g}^{\prime \prime}(s)\right) \boldsymbol{t}(s)\right. \\
& +\left(\kappa_{g}^{\prime \prime \prime}(s)-\kappa_{g}^{\prime}(s)-6 \kappa_{g}^{2}(s) \kappa_{g}^{\prime}(s)-3 \kappa_{g}^{\prime}(s) \tau_{g}^{2}(s)-3 \kappa_{g}(s) \tau_{g}(s) \tau_{g}^{\prime}(s)\right) \boldsymbol{n}(s) \\
& +\left(3 \kappa_{g}^{\prime \prime}(s) \tau_{g}(s)+3 \kappa_{g}^{\prime}(s) \tau_{g}^{\prime}(s)+\kappa_{g}(s) \tau_{g}^{\prime \prime}(s)-\kappa_{g}(s) \tau_{g}(s)-\kappa_{g}^{3}(s) \tau_{g}(s)-\kappa_{g}(s) \tau_{g}^{3}(s)\right) \boldsymbol{b}(s) \\
& \left.+5 \kappa_{g}(s) \kappa_{g}^{\prime}(s) \bar{\gamma}(s), \quad \bar{\gamma}(s)+\frac{1}{\kappa_{g}(s)} \boldsymbol{n}(s)-\frac{\kappa_{g}^{\prime}(s)}{\kappa_{g}^{2}(s) \tau_{g}(s)} \boldsymbol{b}(s) \pm \rho(s) \boldsymbol{e}_{0}\right\rangle \\
& =\frac{1}{\kappa_{g}^{2}(s) \tau_{g}(s)}\left(\kappa_{g}^{\prime \prime \prime}(s) \kappa_{g}(s) \tau_{g}(s)-2 \kappa_{g}(s) \kappa_{g}^{\prime}(s) \tau_{g}^{3}(s)-3 \kappa_{g}^{2}(s) \tau_{g}^{2}(s) \tau_{g}^{\prime}(s)-3 \kappa_{g}^{\prime}(s) \kappa_{g}^{\prime \prime}(s) \tau_{g}(s)\right. \\
& \left.-3 \kappa_{g}^{\prime 2}(s) \tau_{g}^{\prime}(s)-\kappa_{g}(s) \kappa_{g}^{\prime}(s) \tau_{g}^{\prime \prime}(s)\right) \\
& =\frac{\left(\kappa_{g}(s) \kappa_{g}^{\prime \prime}(s) \tau_{g}(s)-2 \kappa_{g}^{\prime 2}(s) \tau_{g}(s)-\kappa_{g}(s) \kappa_{g}^{\prime}(s) \tau_{g}^{\prime}(s)-\kappa_{g}^{2}(s) \tau_{g}^{3}(s)\right)^{\prime}}{\kappa_{g}^{2}(s) \tau_{g}(s)}=0 .
\end{aligned}
$$

This is equivalent to the condition $\left(\left(-1 / \kappa_{g}(s)\right)^{\prime} / \tau_{g}(s)\right)^{\prime}-\tau_{g}(s) / \kappa_{g}(s)=\left(\left(\left(-1 / \kappa_{g}(s)\right)^{\prime} / \tau_{g}(s)\right)^{\prime}-\right.$ $\left.\tau_{g}(s) / \kappa_{g}(s)\right)^{\prime}=0$. Then we have $\kappa_{g}(s) \neq 0, \tau_{g}(s) \neq 0,\left(\left(-1 / \kappa_{g}(s)\right)^{\prime} / \tau_{g}(s)\right)^{\prime}-\tau_{g}(s) / \kappa_{g}(s)=$ $\left(\left(\left(-1 / \kappa_{g}(s)\right)^{\prime} / \tau_{g}(s)\right)^{\prime}-\tau_{g}(s) / \kappa_{g}(s)\right)^{\prime}=0$ and $\boldsymbol{v}=\bar{\gamma}(s)+\boldsymbol{n}(s) / \kappa_{g}(s)-\kappa_{g}^{\prime}(s) \boldsymbol{b}(s) / \kappa_{g}^{2}(s) \tau_{g}(s) \pm$ $\rho(s) \boldsymbol{e}_{0}$. This completes the proof.

Proposition 5.2. Let $\gamma: I \longrightarrow S_{+}^{3}$ be a unit speed curve, then we have the followings:

(1) $h_{\boldsymbol{v}}(s)=0$ if and only if $\boldsymbol{v}=\lambda \bar{\gamma}(s)+\mu \boldsymbol{t}(s)+\xi \boldsymbol{n}(s)+\eta \boldsymbol{b}(s)+(\lambda+2) \boldsymbol{e}_{0}$, where $\lambda, \mu, \xi, \eta \in \mathbb{R}$ and $\mu^{2}+\xi^{2}+\eta^{2}-4 \lambda-4=0$.

(2) $h_{\boldsymbol{v}}(s)=h_{\boldsymbol{v}}^{\prime}(s)=0$ if and only if $\boldsymbol{v}=\left(\left(\xi^{2}+\eta^{2}\right) / 4-1\right) \bar{\gamma}(s)+\xi \boldsymbol{n}+\eta \boldsymbol{b}(s)+\left(\left(\xi^{2}+\eta^{2}\right) / 4+1\right) \boldsymbol{e}_{0}$.

(3) $h_{\boldsymbol{v}}(s)=h_{\boldsymbol{v}}^{\prime}(s)=h_{\boldsymbol{v}}^{\prime \prime}(s)=0$ if and only if

$$
\boldsymbol{v}=\kappa_{g}(s) \xi \bar{\gamma}(s)+\xi \boldsymbol{n}(s) \pm \sqrt{4+4 \kappa_{g}(s) \xi-\xi^{2}} \boldsymbol{b}(s)+\left(\kappa_{g}(s) \xi+2\right) \boldsymbol{e}_{0}
$$


(4) $h_{\boldsymbol{v}}(s)=h_{\boldsymbol{v}}^{\prime}(s)=h_{\boldsymbol{v}}^{\prime \prime}(s)=h_{\boldsymbol{v}}^{\prime \prime \prime}(s)=0$ if and only if $\kappa_{g}^{\prime 2}(s)+\kappa_{g}^{2}(s) \tau_{g}^{2}(s) \neq 0$ and $\boldsymbol{v}=2 \kappa_{g}^{2}(s) \tau_{g}(s) \sigma^{ \pm}(s) \bar{\gamma}(s)+2 \kappa_{g}(s) \tau_{g}(s) \sigma^{ \pm}(s) \boldsymbol{n}(s)-2 \kappa_{g}^{\prime}(s) \sigma^{ \pm}(s) \boldsymbol{b}(s)+\left(2 \kappa_{g}^{2}(s) \tau_{g}(s) \sigma^{ \pm}(s)+2\right) \boldsymbol{e}_{0}$.

(5) $h_{\boldsymbol{v}}(s)=h_{\boldsymbol{v}}^{\prime}(s)=h_{\boldsymbol{v}}^{\prime \prime}(s)=h_{\boldsymbol{v}}^{\prime \prime \prime}(s)=h_{\boldsymbol{v}}^{(4)}(s)=0$ if and only if $\kappa_{g}^{\prime 2}(s)+\kappa_{g}^{2}(s) \tau_{g}^{2}(s) \neq 0$,

$$
\left(\left(\frac{-1}{\kappa_{g}(s)}\right)^{\prime} \frac{1}{\tau_{g}(s)}\right)^{\prime}-\frac{\tau_{g}(s)}{\kappa_{g}(s)}=0
$$

and

$\boldsymbol{v}=2 \kappa_{g}^{2}(s) \tau_{g}(s) \sigma^{ \pm}(s) \bar{\gamma}(s)+2 \kappa_{g}(s) \tau_{g}(s) \sigma^{ \pm}(s) \boldsymbol{n}(s)-2 \kappa_{g}^{\prime}(s) \sigma^{ \pm}(s) \boldsymbol{b}(s)+\left(2 \kappa_{g}^{2}(s) \tau_{g}(s) \sigma^{ \pm}(s)+2\right) \boldsymbol{e}_{0}$.

(6) $h_{\boldsymbol{v}}(s)=h_{\boldsymbol{v}}^{\prime}(s)=h_{\boldsymbol{v}}^{\prime \prime}(s)=h_{\boldsymbol{v}}^{\prime \prime \prime}(s)=h_{\boldsymbol{v}}^{(4)}(s)=h_{\boldsymbol{v}}^{(5)}(s)=0$ if and only if $\kappa_{g}^{\prime 2}(s)+\kappa_{g}^{2}(s) \tau_{g}^{2}(s) \neq 0$,

$$
\left(\left(\frac{-1}{\kappa_{g}(s)}\right)^{\prime} \frac{1}{\tau_{g}(s)}\right)^{\prime}-\frac{\tau_{g}(s)}{\kappa_{g}(s)}=\left\{\left(\left(\frac{-1}{\kappa_{g}(s)}\right)^{\prime} \frac{1}{\tau_{g}(s)}\right)^{\prime}-\frac{\tau_{g}(s)}{\kappa_{g}(s)}\right\}^{\prime}=0
$$

and

$\boldsymbol{v}=2 \kappa_{g}^{2}(s) \tau_{g}(s) \sigma^{ \pm}(s) \bar{\gamma}(s)+2 \kappa_{g}(s) \tau_{g}(s) \sigma^{ \pm}(s) \boldsymbol{n}(s)-2 \kappa_{g}^{\prime}(s) \sigma^{ \pm}(s) \boldsymbol{b}(s)+\left(2 \kappa_{g}^{2}(s) \tau_{g}(s) \sigma^{ \pm}(s)+2\right) \boldsymbol{e}_{0}$.

Proof. (1) Since $\boldsymbol{v} \in L C^{*}$, there exist $\lambda, \mu, \xi, \eta, \omega \in \mathbb{R}$ with $\lambda^{2}+\mu^{2}+\xi^{2}+\eta^{2}-\omega^{2}=0$ such that $\boldsymbol{v}=\lambda \bar{\gamma}(s)+\mu \boldsymbol{t}(s)+\xi \boldsymbol{n}(s)+\eta \boldsymbol{b}(s)+\omega \boldsymbol{e}_{0}$. From $h_{\boldsymbol{v}}(s)=\langle\boldsymbol{\gamma}(s), \boldsymbol{v}\rangle+2=\langle\bar{\gamma}(s)+$ $\left.\boldsymbol{e}_{0}, \lambda \overline{\boldsymbol{\gamma}}(s)+\mu \boldsymbol{t}(s)+\xi \boldsymbol{n}(s)+\eta \boldsymbol{b}(s)+\omega \boldsymbol{e}_{0}\right\rangle+2=\lambda-\omega+2=0$, we have $\omega=2+\lambda$. So $\boldsymbol{v}=$ $\lambda \overline{\boldsymbol{\gamma}}(s)+\mu \boldsymbol{t}(s)+\xi \boldsymbol{n}(s)+\eta \boldsymbol{b}(s)+(2+\lambda) \boldsymbol{e}_{0}$ and $\lambda^{2}+\mu^{2}+\xi^{2}+\eta^{2}-(2+\lambda)^{2}=\mu^{2}+\xi^{2}+\eta^{2}-4 \lambda-4=0$. The converse direction also holds.

(2) Since $h_{\boldsymbol{v}}^{\prime}(s)=\langle\boldsymbol{t}(s), \boldsymbol{v}\rangle, h_{\boldsymbol{v}}(s)=h_{\boldsymbol{v}}^{\prime}(s)=0$ if and only if

$$
h_{\boldsymbol{v}}^{\prime}(s)=\left\langle\boldsymbol{t}(s), \lambda \bar{\gamma}(s)+\mu \boldsymbol{t}(s)+\xi \boldsymbol{n}(s)+\eta \boldsymbol{b}(s)+(2+\lambda) \boldsymbol{e}_{0}\right\rangle=\mu=0 .
$$

By $\lambda^{2}+\xi^{2}+\eta^{2}-(2+\lambda)^{2}=\xi^{2}+\eta^{2}-4 \lambda-4=0$, we have $\lambda=\left(\xi^{2}+\eta^{2}\right) / 4-1$. So, $\boldsymbol{v}=\left(\left(\xi^{2}+\eta^{2}\right) / 4-1\right) \bar{\gamma}(s)+\xi \boldsymbol{n}(s)+\eta \boldsymbol{b}(s)+\left(\left(\xi^{2}+\eta^{2}\right) / 4+1\right) \boldsymbol{e}_{0}$.

(3) Since $h_{\boldsymbol{v}}^{\prime \prime}(s)=\left\langle\kappa_{g}(s) \boldsymbol{n}(s)-\bar{\gamma}(s), \boldsymbol{v}\right\rangle, h_{\boldsymbol{v}}(s)=h_{\boldsymbol{v}}^{\prime}(s)=h_{\boldsymbol{v}}^{\prime \prime}(s)=0$ if and only if

$$
\begin{aligned}
h_{\boldsymbol{v}}^{\prime \prime}(s) & =\left\langle\kappa_{g}(s) \boldsymbol{n}(s)-\bar{\gamma}(s),\left(\frac{\xi^{2}+\eta^{2}}{4}-1\right) \overline{\boldsymbol{\gamma}}(s)+\xi \boldsymbol{n}(s)+\eta \boldsymbol{b}(s)+\left(\frac{\xi^{2}+\eta^{2}}{4}+1\right) \boldsymbol{e}_{0}\right\rangle \\
& =\left(4 \kappa_{g}(s) \xi-\xi^{2}-\eta^{2}+4\right) / 4=0,
\end{aligned}
$$

so that we have $\eta= \pm \sqrt{4+4 \kappa_{g}(s) \xi-\xi^{2}}$ and $\boldsymbol{v}=\kappa_{g}(s) \xi \bar{\gamma}(s)+\xi \boldsymbol{n}(s) \pm \sqrt{4+4 \kappa_{g}(s) \xi-\xi^{2}} \boldsymbol{b}(s)+$ $\left(\kappa_{g}(s) \xi+2\right) \boldsymbol{e}_{0}$.

(4) Since $h_{\boldsymbol{v}}^{\prime \prime \prime}(s)=\left\langle\kappa_{g}^{\prime}(s) \boldsymbol{n}(s)-\left(\kappa_{g}^{2}(s)+1\right) \boldsymbol{t}(s)+\kappa_{g}(s) \tau_{g}(s) \boldsymbol{b}(s), \boldsymbol{v}\right\rangle, h_{\boldsymbol{v}}(s)=h_{\boldsymbol{v}}^{\prime}(s)=h_{\boldsymbol{v}}^{\prime \prime}(s)=$ $h_{\boldsymbol{v}}^{\prime \prime \prime}(s)=0$ if and only if

$$
\begin{aligned}
& h_{\boldsymbol{v}}^{\prime \prime \prime}(s)=\left\langle\kappa_{g}^{\prime}(s) \boldsymbol{n}(s)-\left(\kappa_{g}^{2}(s)+1\right) \boldsymbol{t}(s)+\kappa_{g}(s) \tau_{g}(s) \boldsymbol{b}(s),\right. \\
&\left.\kappa_{g}(s) \xi \bar{\gamma}(s)+\xi \boldsymbol{n}(s) \pm \sqrt{4+4 \kappa_{g}(s) \xi-\xi^{2}} \boldsymbol{b}(s)+\left(\kappa_{g}(s) \xi+2\right) \boldsymbol{e}_{0}\right\rangle \\
&=\kappa_{g}^{\prime}(s) \xi+\kappa_{g}(s) \tau_{g}(s) \eta=\kappa_{g}^{\prime}(s) \xi \pm \kappa_{g}(s) \tau_{g}(s) \sqrt{4+4 \kappa_{g}(s) \xi-\xi^{2}}=0,
\end{aligned}
$$


so that we have $\kappa_{g}^{\prime 2}(s)+\kappa_{g}^{2}(s) \tau_{g}^{2}(s) \neq 0, \xi=2 \kappa_{g}(s) \tau_{g}(s) \sigma^{ \pm}(s)$ and $\boldsymbol{v}=2 \kappa_{g}^{2}(s) \tau_{g}(s) \sigma^{ \pm}(s) \bar{\gamma}(s)+$ $2 \kappa_{g}(s) \tau_{g}(s) \sigma^{ \pm}(s) \boldsymbol{n}(s)-2 \kappa_{g}^{\prime}(s) \sigma^{ \pm}(s) \boldsymbol{b}(s)+\left(2 \kappa_{g}^{2}(s) \tau_{g}(s) \sigma^{ \pm}(s)+2\right) \boldsymbol{e}_{0}$.

(5) Since $h_{\boldsymbol{v}}^{(4)}(s)=\left\langle\left(\kappa_{g}^{\prime \prime}(s)-\kappa_{g}^{3}(s)-\kappa_{g}(s)-\kappa_{g}(s) \tau_{g}^{2}(s)\right) \boldsymbol{n}(s)-3 \kappa_{g}(s) \kappa_{g}^{\prime}(s) \boldsymbol{t}(s)+\left(2 \kappa_{g}^{\prime}(s) \tau_{g}(s)+\right.\right.$ $\left.\left.\kappa_{g}(s) \tau_{g}^{\prime}(s)\right) \boldsymbol{b}(s)+\left(1+\kappa_{g}^{2}(s)\right) \overline{\boldsymbol{\gamma}}(s), \boldsymbol{v}\right\rangle, h_{\boldsymbol{v}}(s)=h_{\boldsymbol{v}}^{\prime}(s)=h_{\boldsymbol{v}}^{\prime \prime}(s)=h_{\boldsymbol{v}}^{\prime \prime \prime}(s)=h_{\boldsymbol{v}}^{(4)}(s)=0$ if and only if

$$
\begin{aligned}
h_{\boldsymbol{v}}^{(4)}(s) & =\left\langle\left(\kappa_{g}^{\prime \prime}(s)-\kappa_{g}^{3}(s)-\kappa_{g}(s)-\kappa_{g}(s) \tau_{g}^{2}(s)\right) \boldsymbol{n}(s)-3 \kappa_{g}(s) \kappa_{g}^{\prime}(s) \boldsymbol{t}(s)\right. \\
& +\left(2 \kappa_{g}^{\prime}(s) \tau_{g}(s)+\kappa_{g}(s) \tau_{g}^{\prime}(s)\right) \boldsymbol{b}(s)+\left(1+\kappa_{g}^{2}(s)\right) \overline{\boldsymbol{\gamma}}(s) \\
& \left.\kappa_{g}(s) \xi \bar{\gamma}(s)+\xi \boldsymbol{n}(s)+\eta \boldsymbol{b}(s)+\left(\kappa_{g}(s) \xi+2\right) \boldsymbol{e}_{0}\right\rangle \\
& =\left(\kappa_{g}^{\prime \prime}(s)-\kappa_{g}(s) \tau_{g}^{2}(s)\right) \xi+\left(2 \kappa_{g}^{\prime}(s) \tau_{g}(s)+\kappa_{g}(s) \tau_{g}^{\prime}(s)\right) \eta=0 .
\end{aligned}
$$

By the above condition, we have the equation $\left(\kappa_{g}^{\prime \prime}(s)-\kappa_{g}(s) \tau_{g}^{2}(s)\right) /\left(2 \kappa_{g}^{\prime}(s) \tau_{g}(s)+\kappa_{g}(s) \tau_{g}^{\prime}(s)\right)=$ $\kappa_{g}^{\prime}(s) / \kappa_{g}(s) \tau_{g}(s)$. It is equivalent to $\left(\left(-1 / \kappa_{g}(s)\right)^{\prime} / \tau_{g}(s)\right)^{\prime}-\tau_{g}(s) / \kappa_{g}(s)=0$. Then we have $\kappa_{g}^{\prime 2}(s)+\kappa_{g}^{2}(s) \tau_{g}^{2}(s) \neq 0,\left(\left(-1 / \kappa_{g}(s)\right)^{\prime} / \tau_{g}(s)\right)^{\prime}-\tau_{g}(s) / \kappa_{g}(s)=0$ and $\boldsymbol{v}=2 \kappa_{g}^{2}(s) \tau_{g}(s) \sigma^{ \pm}(s) \bar{\gamma}(s)+$ $2 \kappa_{g}(s) \tau_{g}(s) \sigma^{ \pm}(s) \boldsymbol{n}(s)-2 \kappa_{g}^{\prime}(s) \sigma^{ \pm}(s) \boldsymbol{b}(s)+\left(2 \kappa_{g}^{2}(s) \tau_{g}(s) \sigma^{ \pm}(s)+2\right) \boldsymbol{e}_{0}$.

(6) Since $h_{\boldsymbol{v}}^{(5)}(s)=\left\langle\left(\kappa_{g}^{4}(s)+2 \kappa_{g}^{2}(s)+\kappa_{g}^{2}(s) \tau_{g}^{2}(s)+1-3 \kappa_{g}^{\prime 2}(s)-4 \kappa_{g}(s) \kappa_{g}^{\prime \prime}(s)\right) \boldsymbol{t}(s)+\left(\kappa_{g}^{\prime \prime \prime}(s)-\right.\right.$ $\left.\kappa_{g}^{\prime}(s)-6 \kappa_{g}^{2}(s) \kappa_{g}^{\prime}(s)-3 \kappa_{g}^{\prime}(s) \tau_{g}^{2}(s)-3 \kappa_{g}(s) \tau_{g}(s) \tau_{g}^{\prime}(s)\right) \boldsymbol{n}(s)+\left(3 \kappa_{g}^{\prime \prime}(s) \tau_{g}(s)+3 \kappa_{g}^{\prime}(s) \tau_{g}^{\prime}(s)+\kappa_{g}(s) \tau_{g}^{\prime \prime}(s)-\right.$ $\left.\left.\kappa_{g}(s) \tau_{g}(s)-\kappa_{g}^{3}(s) \tau_{g}(s)-\kappa_{g}(s) \tau_{g}^{3}(s)\right) \boldsymbol{b}(s)+5 \kappa_{g}(s) \kappa_{g}^{\prime}(s) \bar{\gamma}(s), \boldsymbol{v}\right\rangle, h_{\boldsymbol{v}}(s)=h_{\boldsymbol{v}}^{\prime}(s)=h_{\boldsymbol{v}}^{\prime \prime}(s)=h_{\boldsymbol{v}}^{\prime \prime \prime}(s)=$ $h_{\boldsymbol{v}}^{(4)}(s)=h_{\boldsymbol{v}}^{(5)}(s)=0$ if and only if

$$
\begin{aligned}
h_{\boldsymbol{v}}^{(5)}(s) & =\left\langle\left(\kappa_{g}^{4}(s)+2 \kappa_{g}^{2}(s)+\kappa_{g}^{2}(s) \tau_{g}^{2}(s)+1-3 \kappa_{g}^{\prime 2}(s)-4 \kappa_{g}(s) \kappa_{g}^{\prime \prime}(s)\right) \boldsymbol{t}(s)\right. \\
& +\left(\kappa_{g}^{\prime \prime \prime}(s)-\kappa_{g}^{\prime}(s)-6 \kappa_{g}^{2}(s) \kappa_{g}^{\prime}(s)-3 \kappa_{g}^{\prime}(s) \tau_{g}^{2}(s)-3 \kappa_{g}(s) \tau_{g}(s) \tau_{g}^{\prime}(s)\right) \boldsymbol{n}(s) \\
& +\left(3 \kappa_{g}^{\prime \prime}(s) \tau_{g}(s)+3 \kappa_{g}^{\prime}(s) \tau_{g}^{\prime}(s)+\kappa_{g}(s) \tau_{g}^{\prime \prime}(s)-\kappa_{g}(s) \tau_{g}(s)-\kappa_{g}^{3}(s) \tau_{g}(s)-\kappa_{g}(s) \tau_{g}^{3}(s)\right) \boldsymbol{b}(s) \\
& \left.+5 \kappa_{g}(s) \kappa_{g}^{\prime}(s) \overline{\boldsymbol{\gamma}}(s), \kappa_{g}(s) \xi \bar{\gamma}(s)+\xi \boldsymbol{n}(s)+\eta \boldsymbol{b}(s)+\left(\kappa_{g}(s) \xi+2\right) \boldsymbol{e}_{0}\right\rangle \\
& =\left(\kappa_{g}^{\prime \prime \prime}(s)-\kappa_{g}^{\prime}(s)-\kappa_{g}^{2}(s) \kappa_{g}^{\prime}(s)-3 \kappa_{g}^{\prime}(s) \tau_{g}^{2}(s)-3 \kappa_{g}(s) \tau_{g}(s) \tau_{g}^{\prime}(s)\right) \xi \\
& +\left(3 \kappa_{g}^{\prime \prime}(s) \tau_{g}(s)+3 \kappa_{g}^{\prime}(s) \tau_{g}^{\prime}(s)+\kappa_{g}(s) \tau_{g}^{\prime \prime}(s)-\kappa_{g}(s) \tau_{g}(s)-\kappa_{g}^{3}(s) \tau_{g}(s)-\kappa_{g}(s) \tau_{g}^{3}(s)\right) \eta=0 .
\end{aligned}
$$

By the above condition, we have the equation $\left(\kappa_{g}^{\prime \prime \prime}(s)-\kappa_{g}^{\prime}(s)-\kappa_{g}^{2}(s) \kappa_{g}^{\prime}(s)-3 \kappa_{g}^{\prime}(s) \tau_{g}^{2}(s)-\right.$ $\left.3 \kappa_{g}(s) \tau_{g}(s) \tau_{g}^{\prime}(s)\right) /\left(3 \kappa_{g}^{\prime \prime}(s) \tau_{g}(s)+3 \kappa_{g}^{\prime}(s) \tau_{g}^{\prime}(s)+\kappa_{g}(s) \tau_{g}^{\prime \prime}(s)-\kappa_{g}(s) \tau_{g}(s)-\kappa_{g}^{3}(s) \tau_{g}(s)-\kappa_{g}(s) \tau_{g}^{3}(s)\right)=$ $\kappa_{g}^{\prime}(s) / \kappa_{g}(s) \tau_{g}(s)$. It is equivalent to $\left(\left(-1 / \kappa_{g}(s)\right)^{\prime} / \tau_{g}(s)\right)^{\prime}-\tau_{g}(s) / \kappa_{g}(s)=\left(\left(\left(-1 / \kappa_{g}(s)\right)^{\prime} / \tau_{g}(s)\right)^{\prime}-\right.$ $\left.\tau_{g}(s) / \kappa_{g}(s)\right)^{\prime}=0$. Then we have $\kappa_{g}^{\prime 2}(s)+\kappa_{g}^{2}(s) \tau_{g}^{2}(s) \neq 0,\left(\left(-1 / \kappa_{g}(s)\right)^{\prime} / \tau_{g}(s)\right)^{\prime}-\tau_{g}(s) / \kappa_{g}(s)=$ $\left(\left(\left(-1 / \kappa_{g}(s)\right)^{\prime} / \tau_{g}(s)\right)^{\prime}-\tau_{g}(s) / \kappa_{g}(s)\right)^{\prime}=0$ and $\boldsymbol{v}=2 \kappa_{g}^{2}(s) \tau_{g}(s) \sigma^{ \pm}(s) \bar{\gamma}(s)+2 \kappa_{g}(s) \tau_{g}(s) \sigma^{ \pm}(s) \boldsymbol{n}(s)-$ $2 \kappa_{g}^{\prime}(s) \sigma^{ \pm}(s) \boldsymbol{b}(s)+\left(2 \kappa_{g}^{2}(s) \tau_{g}(s) \sigma^{ \pm}(s)+2\right) \boldsymbol{e}_{0}$. This completes the proof.

According to the assertions of Propositions 5.1 and 5.2, we define an invariant

$$
\kappa_{S}(s)=\left(\left(\frac{-1}{\kappa_{g}(s)}\right)^{\prime} \frac{1}{\tau_{g}(s)}\right)^{\prime}-\frac{\tau_{g}(s)}{\kappa_{g}(s)},
$$

which we call a spherical curvature of $\bar{\gamma}$. We have the following proposition.

Proposition 5.3. For a unit speed curve $\gamma: I \rightarrow S_{+}^{3}$, both of the spherical evolutes $\varepsilon_{\bar{\gamma}}^{ \pm}(s)$ are constant if and only if $\kappa_{S} \equiv 0$.

Proof. $\varepsilon_{\bar{\gamma}}^{\prime \pm}(s)= \pm\left(\kappa_{g}(s) \kappa_{g}^{\prime}(s)\left(2 \kappa_{g}^{\prime 2}(s) \tau_{g}(s)+\kappa_{g}(s) \kappa_{g}^{\prime}(s) \tau_{g}^{\prime}(s)+\kappa_{g}^{2}(s) \tau_{g}^{3}(s)-\kappa_{g}(s) \kappa_{g}^{\prime \prime}(s) \tau_{g}(s)\right)\{\bar{\gamma}(s)+\right.$ $\left.\boldsymbol{n}(s) / \kappa_{g}(s)+\left(1 / \kappa_{g}(s)\right)^{\prime} \boldsymbol{b}(s) / \tau_{g}(s)\right\} /\left(\kappa_{g}^{\prime 2}(s)+\kappa_{g}^{4}(s) \tau_{g}^{2}(s)+\kappa_{g}^{2}(s) \tau_{g}^{2}(s)\right)^{3 / 2}+\left(2 \kappa_{g}^{\prime 2}(s) \tau_{g}(s)+\kappa_{g}(s) \kappa_{g}^{\prime}(s) \tau_{g}^{\prime}(s)+\right.$ $\left.\left.\kappa_{g}^{2}(s) \tau_{g}^{3}(s)-\kappa_{g}(s) \kappa_{g}^{\prime \prime}(s) \tau_{g}(s)\right) \boldsymbol{b}(s) / \kappa_{g}(s) \tau_{g}(s)\left(\kappa_{g}^{\prime 2}(s)+\kappa_{g}^{4}(s) \tau_{g}^{2}(s)+\kappa_{g}^{2}(s) \tau_{g}^{2}(s)\right)^{1 / 2}\right)$. 
On the other hand $\kappa_{S}(s)=\left(\left(-1 / \kappa_{g}(s)\right)^{\prime} / \tau_{g}(s)\right)^{\prime}-\tau_{g}(s) / \kappa_{g}(s)=\left(\kappa_{g}(s) \kappa_{g}^{\prime \prime}(s) \tau_{g}(s)-2 \kappa_{g}^{\prime 2}(s) \tau_{g}(s)-\right.$ $\left.\kappa_{g}(s) \kappa_{g}^{\prime}(s) \tau_{g}^{\prime}(s)-\kappa_{g}^{2}(s) \tau_{g}^{3}(s)\right) / \kappa_{g}^{3}(s) \tau_{g}^{2}(s)=0$. So $\varepsilon_{\bar{\gamma}}^{\prime \pm} \equiv 0$ if and only if $\kappa_{S} \equiv 0$. This completes the proof.

\section{Singularities of ligtcone duals of spherical curves}

In this section we classify the singularities of $\overline{L D}_{\bar{\gamma}}^{ \pm}$and $L D_{\gamma}$ as an application of the unfolding theory of functions. Let $F:\left(\mathbb{R} \times \mathbb{R}^{r},\left(s_{0}, \boldsymbol{x}_{0}\right)\right) \longrightarrow \mathbb{R}$ be a function germ, we call $F$ an $r$-parameter unfolding of $f$, where $f(s)=F_{\boldsymbol{x}_{0}}\left(s, \boldsymbol{x}_{0}\right)$. The discriminant set of $F$ is defined by

$$
D_{F}=\left\{\boldsymbol{x} \in \mathbb{R}^{r} \mid \exists s \in \mathbb{R}, F(s, \boldsymbol{x})=\frac{\partial F}{\partial s}(s, \boldsymbol{x})=0\right\} .
$$

By Propositions 5.1, (2) and 5.2, (2), the discriminant set of $\bar{H}$ and $H$ are

$$
\begin{aligned}
& D_{\bar{H}}=\left\{\bar{\gamma}(s)+u \boldsymbol{n}(s)+v \boldsymbol{b}(s) \pm \sqrt{u^{2}+v^{2}+1} \boldsymbol{e}_{0} \mid s \in I, u, v \in \mathbb{R}\right\}, \\
& D_{H}=\left\{\left(u^{2}+v^{2}-4\right) \bar{\gamma}(s) / 4+u \boldsymbol{n}(s)+v \boldsymbol{b}(s)+\left(u^{2}+v^{2}+4\right) \boldsymbol{e}_{0} / 4 \mid s \in I, u, v \in \mathbb{R}\right\} .
\end{aligned}
$$

These are the lightcone dual surfaces of $\bar{\gamma}$ and the lightcone dual surface of $\gamma$ respectively. Moreover, the both assertions (4) of Propositions 5.1 and 5.2 describe the singularities of the lightcone focal surfaces of $\gamma$ and $\bar{\gamma}$ respectively.

Proposition 6.1. The critical value sets of $L F_{\bar{\gamma}}^{ \pm}$and $L F_{\gamma}^{ \pm}$are give as follows:

$$
\begin{aligned}
C\left(L F_{\bar{\gamma}}^{ \pm}\right)= & \left\{\bar{\gamma}(s)+\frac{1}{\kappa_{g}(s)} \boldsymbol{n}(s)-\frac{\kappa_{g}^{\prime}(s)}{\kappa_{g}^{2}(s) \tau_{g}(s)} \boldsymbol{b}(s) \pm \rho(s) \boldsymbol{e}_{0} \mid s \in I\right\}, \\
C\left(L F_{\gamma}^{ \pm}\right)= & \left\{2 \kappa_{g}^{2}(s) \tau_{g}(s) \sigma^{ \pm}(s) \bar{\gamma}(s)+2 \kappa_{g}(s) \tau_{g}(s) \sigma^{ \pm}(s) \boldsymbol{n}(s)\right. \\
& \left.\quad-2 \kappa_{g}^{\prime}(s) \sigma^{ \pm}(s) \boldsymbol{b}(s)+\left(2 \kappa_{g}^{2}(s) \tau_{g}(s) \sigma^{ \pm}(s)+2\right) \boldsymbol{e}_{0} \mid s \in I\right\} .
\end{aligned}
$$

Then we have the following theorem as a corollary.

Theorem 6.2. Both of the projections of the critical value sets $C\left(L F_{\bar{\gamma}}^{ \pm}\right)$and $C\left(L F_{\gamma}^{ \pm}\right)$in the unit 3-sphere $S_{0}^{3}$ are the images of the spherical evolutes of $\bar{\gamma}$, that is

$$
\tilde{\pi}\left(C\left(L F_{\bar{\gamma}}^{ \pm}\right)\right)=\widetilde{\pi}\left(C\left(L F_{\gamma}^{ \pm}\right)\right)=\left\{\varepsilon_{\bar{\gamma}}^{ \pm}(s) \mid s \in I\right\} .
$$

Proof. We know that

$$
\widetilde{\pi}\left(C\left(L F_{\bar{\gamma}}^{ \pm}\right)\right)=\left\{ \pm\left(\frac{\bar{\gamma}}{\rho(s)}+\frac{\boldsymbol{n}(s)}{\rho(s) \kappa_{g}(s)}-\frac{\kappa_{g}^{\prime}(s) \boldsymbol{b}(s)}{\rho(s) \kappa_{g}^{2}(s) \tau_{g}(s)}\right) \mid s \in I\right\}
$$

and

$$
\widetilde{\pi}\left(C\left(L F_{\gamma}^{ \pm}\right)\right)=\left\{\frac{\kappa_{g}^{2}(s) \tau_{g}(s) \sigma^{ \pm}(s) \bar{\gamma}(s)+\kappa_{g}(s) \tau_{g}(s) \sigma^{ \pm}(s) \boldsymbol{n}(s)-\kappa_{g}^{\prime}(s) \sigma^{ \pm}(s) \boldsymbol{b}(s)}{\kappa_{g}^{2}(s) \tau_{g}(s) \sigma^{ \pm}(s)+1} \mid s \in I\right\} .
$$


By straightforward calculations, we have

$$
\begin{aligned}
& \frac{\kappa_{g}^{2}(s) \tau_{g}(s) \sigma^{ \pm}(s)}{\kappa_{g}^{2}(s) \tau_{g}(s) \sigma^{ \pm}(s)+1} \\
= & \frac{\kappa_{g}^{2}(s) \tau_{g}(s)\left(\kappa_{g}^{2}(s) \tau_{g}(s) \pm \sqrt{\kappa_{g}^{\prime 2}(s)+\kappa_{g}^{2}(s) \tau_{g}^{2}(s)+\kappa_{g}^{4}(s) \tau_{g}^{2}(s)}\right.}{\kappa_{g}^{\prime 2}(s)+\kappa_{g}^{2}(s) \tau_{g}^{2}(s)+\kappa_{g}^{4}(s) \tau_{g}^{2}(s) \pm \kappa_{g}^{2}(s) \tau_{g}(s) \sqrt{\kappa_{g}^{\prime 2}(s)+\kappa_{g}^{2}(s) \tau_{g}^{2}(s)+\kappa_{g}^{4}(s) \tau_{g}^{2}(s)}} \\
= & \frac{ \pm 1}{\sqrt{\kappa_{g}^{\prime 2}(s)+\kappa_{g}^{2}(s) \tau_{g}^{2}(s)+\kappa_{g}^{4}(s) \tau_{g}^{2}(s)}} .
\end{aligned}
$$

Similarly, we can calculate that

$$
\begin{gathered}
\frac{\kappa_{g}(s) \tau_{g}(s) \sigma^{ \pm}(s)}{\kappa_{g}^{2}(s) \tau_{g}(s) \sigma^{ \pm}(s)+1}=\frac{ \pm 1}{\rho(s) \kappa_{g}(s)}, \\
\frac{\kappa_{g}^{\prime}(s) \sigma^{ \pm}(s)}{\kappa_{g}^{2}(s) \tau_{g}(s) \sigma^{ \pm}(s)+1}=\frac{ \pm \kappa_{g}^{\prime}(s)}{\rho(s) \kappa_{g}^{2}(s) \tau_{g}(s)} .
\end{gathered}
$$

So we have

$$
\tilde{\pi}\left(C\left(L F_{\bar{\gamma}}^{ \pm}\right)\right)=\tilde{\pi}\left(C\left(L F_{\gamma}^{ \pm}\right)\right)=\left\{\varepsilon_{\bar{\gamma}}^{ \pm}(s) \mid s \in I\right\} .
$$

This completes the proof.

Inspired by Propositons 5.1, 5.2 and Theorem 6.2, we define the following set:

$$
D_{F}^{\ell}=\left\{\boldsymbol{x} \in \mathbb{R}^{r} \mid \exists s \in \mathbb{R}, F(s, \boldsymbol{x})=\frac{\partial F}{\partial s}(s, \boldsymbol{x})=\cdots=\frac{\partial^{\ell} F}{\partial s^{\ell}}(s, \boldsymbol{x})=0\right\},
$$

which is called a discriminant set of order $\ell$. Of course, $D_{F}^{1}=D_{F}$. In order to understand the geometric properties of the discriminant set of order $\ell$, we introduce an equivalence relation among the unfoldings of functions. Let $F$ and $G$ be $r$-parameter unfoldings of $f(s)$ and $g(s)$, respectively. We say that $F$ and $G$ are $P$ - $\mathcal{R}$-equivalent if there exists a diffeomorphism germ $\Phi:\left(\mathbb{R} \times \mathbb{R}^{r},\left(s_{0}, \boldsymbol{x}_{0}\right)\right) \longrightarrow\left(\mathbb{R} \times \mathbb{R}^{r},\left(s_{0}^{\prime}, \boldsymbol{x}_{0}^{\prime}\right)\right)$ of the form $\Phi(s, \boldsymbol{x})=\left(\Phi_{1}(s, \boldsymbol{x}), \phi(\boldsymbol{x})\right)$ such that $G \circ \Phi=F$. By straightforward calculations, we have the following proposition.

Proposition 6.3. Let $F$ and $G$ be r-parameter unfoldings of $f(s)$ and $g(s)$, respectively. If $F$ and $G$ are $P$ - $\mathcal{R}$-equivalent by a diffeomorphism germ $\Phi:\left(\mathbb{R} \times \mathbb{R}^{r},\left(s_{0}, \boldsymbol{x}_{0}\right)\right) \longrightarrow\left(\mathbb{R} \times \mathbb{R}^{r},\left(s_{0}^{\prime}, \boldsymbol{x}_{0}^{\prime}\right)\right)$ of the form $\Phi(s, \boldsymbol{x})=\left(\Phi_{1}(s, \boldsymbol{x}), \phi(\boldsymbol{x})\right)$, then $\phi\left(D_{F}^{\ell}\right)=D_{G}^{\ell}$ as set germs.

By Propositions 5.1 and 5.2, we have the following proposition.

Proposition 6.4. Under the same notations as in the previous paragraphs, we have

$$
\begin{aligned}
& D_{\bar{H}}=D_{\bar{H}}^{1}=\text { Image } \overline{L D_{\bar{\gamma}}^{ \pm}}, D_{\bar{H}}^{2}=\text { Image } L F_{\bar{\gamma}}^{ \pm}, \tilde{\pi}\left(D_{\bar{H}}^{3}\right)=\text { Image } \varepsilon_{\bar{\gamma}}^{ \pm}, \\
& D_{H}=D_{H}^{1}=\text { Image } L D_{\gamma}, D_{H}^{2}=\text { Image } L F_{\gamma}^{ \pm}, \widetilde{\pi}\left(D_{H}^{3}\right)=\text { Image } \varepsilon_{\bar{\gamma}}^{ \pm} .
\end{aligned}
$$

For a function $f(s)$, we say that $f$ has $A_{k}$-singularity at $s_{0}$ if $f^{(p)}\left(s_{0}\right)=0$ for all $1 \leq p \leq k$ and $f^{(k+1)}\left(s_{0}\right) \neq 0$. Let $F$ be an $r$-parameter unfolding of $f$ and $f$ has $A_{k}$-singularity $(k \geq 1)$ at $s_{0}$. We denote the $(k-1)$-jet of the partial derivative $\partial F / \partial x_{i}$ at $s_{0}$ as

$$
j^{(k-1)}\left(\frac{\partial F}{\partial x_{i}}\left(s, \boldsymbol{x}_{0}\right)\right)\left(s_{0}\right)=\sum_{j=1}^{k-1} \alpha_{j i}\left(s-s_{0}\right)^{j}, \quad(i=1, \cdots, r) .
$$


If the rank of $k \times r$ matrix $\left(\alpha_{0 i}, \alpha_{j i}\right)$ is $k(k \leq r)$, then $F$ is called a versal unfolding of $f$, where $\alpha_{0 i}=\partial F / \partial x_{i}\left(s_{0}, \boldsymbol{x}_{0}\right)$. We have the following classification theorem of versal unfoldings [3, Page 149, 6.6].

Theorem 6.5. Let $F:\left(\mathbb{R} \times \mathbb{R}^{r},\left(s_{0}, \boldsymbol{x}_{0}\right)\right) \longrightarrow \mathbb{R}$ be an $r$-parameter unfolding of $f$ which has $A_{k}$-singularity at $s_{0}$. Suppose $F$ is a versal unfolding of $f$, then $F$ is $P$ - $\mathcal{R}$-equivalent to one of the following unfoldings:
(a) $k=1 ; \pm s^{2}+x_{1}$,
(b) $k=2 ; s^{3}+x_{1}+s x_{2}$,
(c) $k=3 ; \pm s^{4}+x_{1}+s x_{2}+s^{2} x_{3}$,
(d) $k=4 ; s^{5}+x_{1}+s x_{2}+s^{2} x_{3}+s^{3} x_{4}$.

We have the following classification result as a corollary of the above theorem.

Corollary 6.6. Let $F:\left(\mathbb{R} \times \mathbb{R}^{r},\left(s_{0}, \boldsymbol{x}_{0}\right)\right) \longrightarrow \mathbb{R}$ be an $r$-parameter unfolding of $f$ which has $A_{k}$-singularity at $s_{0}$. Suppose $F$ is a versal unfolding of $f$, then we have the following assertions:

(a) If $k=1$, then $D_{F}$ is diffeomorphic to $\{0\} \times \mathbb{R}^{r-1}$ and $D_{F}^{2}=\emptyset$.

(b) If $k=2$, then $D_{F}$ is diffeomorphic to $C(2,3) \times \mathbb{R}^{r-2}, D_{F}^{2}$ is diffeomorphic to $\{\mathbf{0}\} \times \mathbb{R}^{r-2}$ and $D_{F}^{3}=\emptyset$.

(c) If $k=3$, then $D_{F}$ is diffeomorphic to $S W \times \mathbb{R}^{r-3}, D_{F}^{2}$ is diffeomorphic to $C(2,3,4) \times \mathbb{R}^{r-3}$, $D_{F}^{3}$ is diffeomorphic to $\{\mathbf{0}\} \times \mathbb{R}^{r-3}$ and $D_{F}^{4}=\emptyset$.

(d) If $k=4$, then $D_{F}$ is locally diffeomorphic to $B F \times \mathbb{R}^{r-4}, D_{F}^{2}$ is diffeomorphic to $C(B F) \times$ $\mathbb{R}^{r-4}, D_{F}^{3}$ is diffeomorphic to $C(2,3,4,5) \times \mathbb{R}^{r-4}, D_{F}^{4}$ is diffeomorphic to $\{\mathbf{0}\} \times \mathbb{R}^{r-4}$ and $D_{F}^{5}=\emptyset$.

We remark that all of diffeomorphisms in the above assertions are diffeomorphism germs.

Here, we respectively call $C(2,3)=\left\{\left(x_{1}, x_{2}\right) \mid x_{1}=u^{2}, x_{2}=u^{3}\right\}$ a $(2,3)$-cusp, $C(2,3,4)=$ $\left\{\left(x_{1}, x_{2}, x_{3}\right) \mid x_{1}=u^{2}, x_{2}=u^{3}, x_{3}=u^{4}\right\}$ a $(2,3,4)$-cusp, $C(2,3,4,5)=\left\{\left(x_{1}, x_{2}, x_{3}, x_{4}\right) \mid x_{1}=\right.$ $\left.u^{2}, x_{2}=u^{3}, x_{3}=u^{4}, x_{4}=u^{5}\right\}$ a $(2,3,4,5)$-cusp, $S W=\left\{\left(x_{1}, x_{2}, x_{3}\right) \mid x_{1}=3 u^{4}+u^{2} v, x_{2}=\right.$ $\left.4 u^{3}+2 u v, x_{3}=v\right\}$ a swallow tail, $B F=\left\{\left(x_{1}, x_{2}, x_{3} . x_{4}\right) \mid x_{1}=5 u^{4}+3 v u^{2}+2 w u, x_{2}=\right.$ $\left.4 u^{5}+2 v u^{3}+w u^{2}, x_{3}=u, x_{4}=v\right\}$ a butterfly and $C(B F)=\left\{\left(x_{1}, x_{2}, x_{3}, x_{4}\right) \mid x_{1}=6 u^{5}+u^{3} v, x_{2}=\right.$ $\left.25 u^{4}+9 u^{2} v, x_{3}=10 u^{3}+3 u v, x_{4}=v\right\}$ a $c$-butterfly (i.e., the critical value set of the butterfly).

We have the following key propositions on $H$ and $\bar{H}$.

Proposition 6.7. If $\bar{h}_{\boldsymbol{v}_{\mathbf{0}}}$ has $A_{k}$-singularity $(k=1,2,3,4)$ at $s_{0}$, then $\bar{H}$ is a versal unfolding of $\bar{h}_{v_{0}}$.

Proof. For $\boldsymbol{v} \in L C^{*}$, we have $\boldsymbol{v}=\left( \pm\left(v_{1}^{2}+v_{2}^{2}+v_{3}^{2}+v_{4}^{2}\right)^{1 / 2}, v_{1}, v_{2}, v_{3}, v_{4}\right)$. We denote that $\bar{\gamma}(s)=\left(0, x_{1}(s), x_{2}(s), x_{3},(s) x_{4}(s)\right)$. Then

$$
\bar{H}(s, \boldsymbol{v})=\langle\bar{\gamma}(s), \boldsymbol{v}\rangle-1=x_{1}(s) v_{1}+x_{2}(s) v_{2}+x_{3}(s) v_{3}+x_{4}(s) v_{4}-1 .
$$

Thus we have

$$
\begin{gathered}
\frac{\partial \bar{H}}{\partial v_{1}}(s, \boldsymbol{v})=x_{1}(s), \frac{\partial \bar{H}}{\partial v_{2}}(s, \boldsymbol{v})=x_{2}(s), \frac{\partial \bar{H}}{\partial v_{3}}(s, \boldsymbol{v})=x_{3}(s), \frac{\partial \bar{H}}{\partial v_{4}}(s, \boldsymbol{v})=x_{4}(s), \\
\frac{\partial^{2} \bar{H}}{\partial s \partial v_{1}}(s, \boldsymbol{v})=x_{1}^{\prime}(s), \frac{\partial^{2} \bar{H}}{\partial s \partial v_{2}}(s, \boldsymbol{v})=x_{2}^{\prime}(s), \frac{\partial^{2} \bar{H}}{\partial s \partial v_{3}}(s, \boldsymbol{v})=x_{3}^{\prime}(s), \frac{\partial^{2} \bar{H}}{\partial s \partial v_{4}}(s, \boldsymbol{v})=x_{4}^{\prime}(s),
\end{gathered}
$$




$$
\begin{gathered}
\frac{\partial^{3} \bar{H}}{\partial s^{2} \partial v_{1}}(s, \boldsymbol{v})=x_{1}^{\prime \prime}(s), \frac{\partial^{3} \bar{H}}{\partial s^{2} \partial v_{2}}(s, \boldsymbol{v})=x_{2}^{\prime \prime}(s), \frac{\partial^{3} \bar{H}}{\partial s^{2} \partial v_{3}}(s, \boldsymbol{v})=x_{3}^{\prime \prime}(s), \frac{\partial^{3} \bar{H}}{\partial s^{2} \partial v_{4}}(s, \boldsymbol{v})=x_{4}^{\prime \prime}(s), \\
\frac{\partial^{4} \bar{H}}{\partial s^{3} \partial v_{1}}(s, \boldsymbol{v})=x_{1}^{\prime \prime \prime}(s), \frac{\partial^{4} \bar{H}}{\partial s^{3} \partial v_{2}}(s, \boldsymbol{v})=x_{2}^{\prime \prime \prime}(s), \frac{\partial^{4} \bar{H}}{\partial s^{3} \partial v_{3}}(s, \boldsymbol{v})=x_{3}^{\prime \prime \prime}(s), \frac{\partial^{4} \bar{H}}{\partial s^{3} \partial v_{4}}(s, \boldsymbol{v})=x_{4}^{\prime \prime \prime}(s) .
\end{gathered}
$$

For a fixed point $\boldsymbol{v}_{0}=\left(v_{00}, v_{01}, v_{02}, v_{03}, v_{04}\right)$, the 3 -jet of $\partial \bar{H} / \partial v_{i}\left(s, \boldsymbol{v}_{0}\right)(i=1,2,3,4)$ at $s_{0}$ is $j^{(3)} \frac{\partial \bar{H}}{\partial v_{i}}\left(s, \boldsymbol{v}_{0}\right)\left(s_{0}\right)=x_{i}^{\prime}\left(s_{0}\right)\left(s-s_{0}\right)+x_{i}^{\prime \prime}\left(s_{0}\right)\left(s-s_{0}\right)^{2} / 2+x_{i}^{\prime \prime \prime}\left(s_{0}\right)\left(s-s_{0}\right)^{3} / 6, \quad(i=1,2,3,4)$.

It is enough to show that the rank of the matrix $\mathrm{A}$ is 4 , where

$$
A=\left(\begin{array}{cccc}
x_{1}\left(s_{0}\right) & x_{2}\left(s_{0}\right) & x_{3}\left(s_{0}\right) & x_{4}\left(s_{0}\right) \\
x_{1}^{\prime}\left(s_{0}\right) & x_{2}^{\prime}\left(s_{0}\right) & x_{3}^{\prime}\left(s_{0}\right) & x_{4}^{\prime}\left(s_{0}\right) \\
x_{1}^{\prime \prime}\left(s_{0}\right) & x_{2}^{\prime \prime}\left(s_{0}\right) & x_{3}^{\prime \prime}\left(s_{0}\right) & x_{4}^{\prime \prime}\left(s_{0}\right) \\
x_{1}^{\prime \prime \prime}\left(s_{0}\right) & x_{2}^{\prime \prime \prime}\left(s_{0}\right) & x_{3}^{\prime \prime \prime}\left(s_{0}\right) & x_{4}^{\prime \prime \prime}\left(s_{0}\right)
\end{array}\right)
$$

Then we have

$$
\operatorname{det} A=\left\langle\boldsymbol{e}_{0} \wedge \bar{\gamma}\left(s_{0}\right) \wedge \bar{\gamma}^{\prime}\left(s_{0}\right) \wedge \bar{\gamma}^{\prime \prime}\left(s_{0}\right), \bar{\gamma}^{\prime \prime \prime}\left(s_{0}\right)\right\rangle=-\kappa_{g}^{2}\left(s_{0}\right) \tau_{g}\left(s_{0}\right) \neq 0
$$

So the rank of $A$ is 4 , this completes the proof.

Proposition 6.8. If $h_{v_{\mathbf{0}}}$ has $A_{k^{-}}$-singularity $(k=1,2,3,4)$ at $s_{0}$, then $H$ is a versal unfolding of $h_{v_{0}}$.

Proof. For $\boldsymbol{v} \in L C^{*}$, we have $\boldsymbol{v}=\left(v_{0}, v_{1}, v_{2}, v_{3}, v_{4}\right)=\left( \pm\left(v_{1}^{2}+v_{2}^{2}+v_{3}^{2}+v_{4}^{2}\right)^{1 / 2}, v_{1}, v_{2}, v_{3}, v_{4}\right)$. We denote that $\bar{\gamma}(s)=\left(1, x_{1}(s), x_{2}(s), x_{3},(s) x_{4}(s)\right)$. Then we have

$$
H(s, \boldsymbol{v})=\langle\gamma(s), \boldsymbol{v}\rangle+2=\mp\left(v_{1}^{2}+v_{2}^{2}+v_{3}^{2}+v_{4}^{2}\right)^{1 / 2}+x_{1}(s) v_{1}+x_{2}(s) v_{2}+x_{3}(s) v_{3}+x_{4}(s) v_{4}+2 .
$$

Thus we have

$$
\begin{gathered}
\frac{\partial H}{\partial v_{1}}(s, \boldsymbol{v})=-v_{1} / v_{0}+x_{1}(s), \frac{\partial H}{\partial v_{2}}(s, \boldsymbol{v})=-v_{2} / v_{0}+x_{2}(s), \\
\frac{\partial H}{\partial v_{3}}(s, \boldsymbol{v})=-v_{3} / v_{0}+x_{3}(s), \frac{\partial H}{\partial v_{4}}(s, \boldsymbol{v})=-v_{4} / v_{0}+x_{4}(s), \\
\frac{\partial^{2} H}{\partial s \partial v_{1}}(s, \boldsymbol{v})=x_{1}^{\prime}(s), \frac{\partial^{2} H}{\partial s \partial v_{2}}(s, \boldsymbol{v})=x_{2}^{\prime}(s), \frac{\partial^{2} H}{\partial s \partial v_{3}}(s, \boldsymbol{v})=x_{3}^{\prime}(s), \frac{\partial^{2} H}{\partial s \partial v_{4}}(s, \boldsymbol{v})=x_{4}^{\prime}(s), \\
\frac{\partial^{3} H}{\partial s^{2} \partial v_{1}}(s, \boldsymbol{v})=x_{1}^{\prime \prime}(s), \frac{\partial^{3} H}{\partial s^{2} \partial v_{2}}(s, \boldsymbol{v})=x_{2}^{\prime \prime}(s), \frac{\partial^{3} H}{\partial s^{2} \partial v_{3}}(s, \boldsymbol{v})=x_{3}^{\prime \prime}(s), \frac{\partial^{3} H}{\partial s^{2} \partial v_{4}}(s, \boldsymbol{v})=x_{4}^{\prime \prime}(s), \\
\frac{\partial^{4} H}{\partial s^{3} \partial v_{1}}(s, \boldsymbol{v})=x_{1}^{\prime \prime \prime}(s), \frac{\partial^{4} H}{\partial s^{3} \partial v_{2}}(s, \boldsymbol{v})=x_{2}^{\prime \prime \prime}(s), \frac{\partial^{4} H}{\partial s^{3} \partial v_{3}}(s, \boldsymbol{v})=x_{3}^{\prime \prime \prime}(s), \frac{\partial^{4} H}{\partial s^{3} \partial v_{4}}(s, \boldsymbol{v})=x_{4}^{\prime \prime \prime}(s) .
\end{gathered}
$$

For a fixed $\boldsymbol{v}_{0}=\left(v_{00}, v_{01}, v_{02}, v_{03}, v_{04}\right)$, the 3 -jet of $\partial H / \partial v_{i}\left(s, \boldsymbol{v}_{0}\right)(i=1,2,3,4)$ at $s_{0}$ is

$$
j^{(3)} \frac{\partial H}{\partial v_{i}}\left(s, \boldsymbol{v}_{0}\right)\left(s_{0}\right)=x_{i}^{\prime}\left(s_{0}\right)\left(s-s_{0}\right)+x_{i}^{\prime \prime}\left(s_{0}\right)\left(s-s_{0}\right)^{2} / 2+x_{i}^{\prime \prime \prime}\left(s_{0}\right)\left(s-s_{0}\right)^{3} / 6, \quad(i=1,2,3,4) .
$$


It is enough to show that the rank of the matrix B is three, where

$$
B=\left(\begin{array}{cccc}
-v_{01} / v_{00}+x_{1}\left(s_{0}\right) & -v_{02} / v_{00}+x_{2}\left(s_{0}\right) & -v_{03} / v_{00}+x_{3}\left(s_{0}\right) & -v_{04} / v_{00}+x_{4}\left(s_{0}\right) \\
x_{1}^{\prime}\left(s_{0}\right) & x_{2}^{\prime}\left(s_{0}\right) & x_{3}^{\prime}\left(s_{0}\right) & x_{4}^{\prime}\left(s_{0}\right) \\
x_{1}^{\prime \prime}\left(s_{0}\right) & x_{2}^{\prime \prime}\left(s_{0}\right) & x_{3}^{\prime \prime}\left(s_{0}\right) & x_{4}^{\prime \prime}\left(s_{0}\right) \\
x_{1}^{\prime \prime \prime}\left(s_{0}\right) & x_{2}^{\prime \prime \prime}\left(s_{0}\right) & x_{3}^{\prime \prime \prime}\left(s_{0}\right) & x_{4}^{\prime \prime \prime}\left(s_{0}\right)
\end{array}\right) .
$$

By straightforward calculations, we have

$\operatorname{det} B=\left\langle\boldsymbol{e}_{0} \wedge \overline{\boldsymbol{\gamma}}^{\prime}\left(s_{0}\right) \wedge \overline{\boldsymbol{\gamma}}^{\prime \prime}\left(s_{0}\right) \wedge \overline{\boldsymbol{\gamma}}^{\prime \prime \prime}\left(s_{0}\right), \boldsymbol{v}_{0}\right\rangle / v_{00}+\left\langle\boldsymbol{e}_{0} \wedge \overline{\boldsymbol{\gamma}}\left(s_{0}\right) \wedge \overline{\boldsymbol{\gamma}}^{\prime}\left(s_{0}\right) \wedge \overline{\boldsymbol{\gamma}}^{\prime \prime}\left(s_{0}\right), \overline{\boldsymbol{\gamma}}^{\prime \prime \prime}\left(s_{0}\right)\right\rangle$ $=\left\langle\kappa_{g}^{2}\left(s_{0}\right) \tau_{g}\left(s_{0}\right) \bar{\gamma}\left(s_{0}\right), \boldsymbol{v}_{0}\right\rangle / v_{00}-\left\langle\kappa_{g}^{\prime}\left(s_{0}\right) \boldsymbol{b}\left(s_{0}\right), \boldsymbol{v}_{0}\right\rangle / v_{00}+\left\langle\kappa_{g}\left(s_{0}\right) \tau_{g}\left(s_{0}\right) \boldsymbol{n}\left(s_{0}\right), \boldsymbol{v}_{0}\right\rangle / v_{00}-\kappa_{g}^{2}\left(s_{0}\right) \tau_{g}\left(s_{0}\right)$. In this case, $h_{\boldsymbol{v}_{\mathbf{0}}}(s)$ has $A_{4}$-singularity, then we have

$$
\begin{gathered}
\boldsymbol{v}_{0}=2 \kappa_{g}^{2}\left(s_{0}\right) \tau_{g}\left(s_{0}\right) \sigma^{ \pm}\left(s_{0}\right) \bar{\gamma}\left(s_{0}\right)+2 \kappa_{g}\left(s_{0}\right) \tau_{g}\left(s_{0}\right) \sigma^{ \pm}\left(s_{0}\right) \boldsymbol{n}\left(s_{0}\right)-2 \kappa_{g}^{\prime}\left(s_{0}\right) \sigma^{ \pm}\left(s_{0}\right) \boldsymbol{b}\left(s_{0}\right) \\
+\left(2 \kappa_{g}^{2}\left(s_{0}\right) \tau_{g}\left(s_{0}\right) \sigma^{ \pm}\left(s_{0}\right)+2\right) \boldsymbol{e}_{0} .
\end{gathered}
$$

Moreover we have

$$
v_{00}=2 \kappa_{g}^{2}\left(s_{0}\right) \tau_{g}\left(s_{0}\right) \sigma^{ \pm}\left(s_{0}\right)+2 .
$$

Therefore by calculation, we have

$$
\operatorname{det} B= \pm \frac{\kappa_{g}^{2}\left(s_{0}\right) \tau_{g}^{2}\left(s_{0}\right)+\kappa_{g}^{\prime 2}\left(s_{0}\right)}{\sqrt{\kappa_{g}^{\prime 2}\left(s_{0}\right)+\kappa_{g}^{2}\left(s_{0}\right) \tau_{g}^{2}\left(s_{0}\right)+\kappa_{g}^{4}\left(s_{0}\right) \tau_{g}^{2}\left(s_{0}\right)} \pm \kappa_{g}^{2}\left(s_{0}\right) \tau_{g}\left(s_{0}\right)} \neq 0
$$

So the rank of $B$ is 4 . This completes the proof.

We have the following theorem:

Theorem 6.9. Let $\gamma: I \longrightarrow S_{+}^{3}$ be a unit speed curve.

(A) For each one of the lightcone duals $\overline{L D}_{\bar{\gamma}}^{ \pm}$of $\bar{\gamma}$, we have the following assertions:

(1) Each one of the lightcone duals $\overline{L D}_{\bar{\gamma}}^{ \pm}$of $\bar{\gamma}$ is locally diffeomorphic to $C(2,3) \times \mathbb{R}^{2}$ at $\left(s_{0}, u_{0}, v_{0}\right)$ if and only if

$$
\kappa_{g}\left(s_{0}\right) \neq 0, u_{0}=\frac{1}{\kappa_{g}\left(s_{0}\right)} \text { and } v_{0} \neq\left(\frac{1}{\kappa_{g}\left(s_{0}\right)}\right)^{\prime} \frac{1}{\tau_{g}\left(s_{0}\right)} .
$$

In this case, each one of $L F_{\bar{\gamma}}^{ \pm}$is non-singular and each one of Images $\varepsilon_{\bar{\gamma}}^{ \pm}$is empty.

(2) Each one of the lightcone duals $\overline{L D}_{\bar{\gamma}}^{ \pm}$of $\bar{\gamma}$ is locally diffeomorphic to $S W \times \mathbb{R}$ at $\left(s_{0}, u_{0}, v_{0}\right)$ if and only if

$$
\kappa_{g}\left(s_{0}\right) \neq 0, \tau_{g}\left(s_{0}\right) \neq 0, u_{0}=\frac{1}{\kappa_{g}\left(s_{0}\right)}, v_{0}=\left(\frac{1}{\kappa_{g}\left(s_{0}\right)}\right)^{\prime} \frac{1}{\tau_{g}\left(s_{0}\right)} \text { and } \kappa_{S}\left(s_{0}\right) \neq 0 .
$$

In this case, each one of $L F_{\bar{\gamma}}^{ \pm}$is locally diffeomorphic to $C(2,3,4) \times \mathbb{R}$ and each one of Images $\varepsilon_{\bar{\gamma}}^{ \pm}$ is a regular curve.

(3) Each one of the lightcone duals $\overline{L D}_{\bar{\gamma}}^{ \pm}$of $\bar{\gamma}$ is locally diffeomorphic to BF at $\left(s_{0}, u_{0}, v_{0}\right)$ if and only if

$$
\kappa_{g}\left(s_{0}\right) \neq 0, \tau_{g}\left(s_{0}\right) \neq 0, u_{0}=\frac{1}{\kappa_{g}\left(s_{0}\right)}, v_{0}=\left(\frac{1}{\kappa_{g}\left(s_{0}\right)}\right)^{\prime} \frac{1}{\tau_{g}\left(s_{0}\right)}, \kappa_{S}\left(s_{0}\right)=0 \text { and } \kappa_{S}^{\prime}\left(s_{0}\right) \neq 0 .
$$


In this case, each one of $L F_{\bar{\gamma}}^{ \pm}$is locally diffeomorphic to $C(B F) \times \mathbb{R}$ and each one of Images $\varepsilon_{\bar{\gamma}}^{ \pm}$ is locally diffeomorphic to the projection of the $C(2,3,4,5)$-cusp.

(B) For the lightcone dual $L D_{\gamma}$ of $\gamma$, we have the following assertions:

(1) The lightcone dual $L D_{\gamma}$ of $\boldsymbol{\gamma}$ is locally diffeomorphic to $C(2,3) \times \mathbb{R}^{2}$ at $\left(s_{0}, u_{0}, v_{0}\right)$ if and only if

$$
u_{0} \neq 2 \kappa_{g}\left(s_{0}\right) \tau_{g}\left(s_{0}\right) \sigma^{ \pm}\left(s_{0}\right) \text { and } v_{0}= \pm \sqrt{4+4 \kappa_{g}\left(s_{0}\right) u_{0}-u_{0}^{2}} .
$$

In this case, each one of $L F_{\gamma}^{ \pm}$is non-singular and each one of Images $\boldsymbol{\varepsilon}_{\bar{\gamma}}^{ \pm}$is empty.

(2) The lightcone dual $L D_{\gamma}$ of $\boldsymbol{\gamma}$ is locally diffeomorphic to $S W \times \mathbb{R}$ at $\left(s_{0}, u_{0}, v_{0}\right)$ if and only if

$$
\kappa_{g}^{\prime 2}\left(s_{0}\right)+\kappa_{g}^{2}\left(s_{0}\right) \tau_{g}^{2}\left(s_{0}\right) \neq 0, u_{0}=2 \kappa_{g}\left(s_{0}\right) \tau_{g}\left(s_{0}\right) \sigma^{ \pm}\left(s_{0}\right), v_{0}=-2 \kappa_{g}^{\prime}\left(s_{0}\right) \sigma^{ \pm}\left(s_{0}\right) \text { and } \kappa_{S}\left(s_{0}\right) \neq 0 .
$$

In this case, each one of $L F_{\gamma}^{ \pm}$is locally diffeomorphic to $C(2,3,4) \times \mathbb{R}$ and each one of Images $\varepsilon_{\bar{\gamma}}^{ \pm}$ is a regular curve.

(3) The lightcone dual $L D_{\gamma}$ of $\gamma$ is locally diffeomorphic to $B F$ at $\left(s_{0}, u_{0}, v_{0}\right)$ if and only if

$$
\begin{aligned}
& \kappa_{g}^{\prime 2}\left(s_{0}\right)+\kappa_{g}^{2}\left(s_{0}\right) \tau_{g}^{2}\left(s_{0}\right) \neq 0, u_{0}=2 \kappa_{g}\left(s_{0}\right) \tau_{g}\left(s_{0}\right) \sigma^{ \pm}\left(s_{0}\right), v_{0}=-2 \kappa_{g}^{\prime}\left(s_{0}\right) \sigma^{ \pm}\left(s_{0}\right) \text {, } \\
& \kappa_{S}\left(s_{0}\right)=0 \text { and } \kappa_{S}^{\prime}\left(s_{0}\right) \neq 0 \text {. }
\end{aligned}
$$

In this case, each one of $L F_{\gamma}^{ \pm}$is locally diffeomorphic to $C(B F) \times \mathbb{R}$ and each one of Images $\varepsilon_{\bar{\gamma}}^{ \pm}$ is locally diffeomorphic to the projection of the $C(2,3,4,5)$-cusp.

Proof. By Propositions 5.1 and 5.2, the discriminant sets of $\bar{H}$ and $H$ are the lightcone duals of $\bar{\gamma}$ and $\boldsymbol{\gamma}$ respectively. By Propositions 5.1 and 5.2, both of $\bar{h}_{\boldsymbol{v}_{0}}$ and $h_{\boldsymbol{v}_{0}}$ have $A_{k}$ singularities $(k=1,2,3,4)$ respectively if and only if the above conditions on the geodesic curvatures and geodesic torsions hold. By Propositions 6.7 and $6.8, \bar{H}$ and $H$ are versal unfoldings of $\bar{h}_{\boldsymbol{v}_{0}}$ and $h_{\boldsymbol{v}_{0}}$ at any point $s_{0} \in I$ respectively. We apply Corollary 6.6, so that we have the above assertions.

\section{References}

[1] V. I. Arnol'd, S. M. Gusein-Zade and A. N. Varchenko, Singularities of Differentiable Maps vol. I. Birkhäuser, 1986.

[2] J. W. Bruce and M. C. Romero-Fuster, Duality and projections of curves and surfaces in 3-space, Quart. J. Math. Oxford Ser. 42 (1991), 433-441.

[3] J. W. Bruce, P. J. Gibline, Curves and singularities(2nd.ed.), Cambridge Univ. Press (1992).

[4] L. Chen and S. Izumiya, A mandala of Legendrian dualities for pseudo-spheres in semiEuclidean space, Proceedings of the Japan Academy, 85 Ser. A, (2009), 49-54.

[5] S. Izumiya, Lengendrian dualities and spacelike hypersurfaces in the lightcone, Mosc. Math. J. 9 (2009) 325-357.

[6] S. Izumiya, Differential Geometry from the viewpoint of Lagrangian or Legendrian singularity theory, in Singularity Theory (ed., D. Chéniot et al), World Scientific (2007), 241-275. 
[7] S. Izumiya, Y. Jiang and D.-H. Pei, Lightcone dualities for curves in the sphere, The Quart. J. Math. (2012)

[8] J. J. Koenderink, Solid shape, The MIT Press (1990).

[9] H. L. Liu, S. D. Jung, Hypersurfaces in lightlike cone, J. Geom. Phys. 58 (2008), 913-922.

[10] T. Nagai, The Gauss map of a hypersurface in Euclidean sphere and the spherical Legendrian duality, Topology and its Applications, 159 (2012), 545-554.

[11] I. R. Porteous, Some remrks on duality in $S^{3}$, Geometry and topology of caustics, Banach Center Publ. 50, Polish Acad. Sci., Warsaw, (2004), 217-226.

[12] M. C. Romero Fuster, Sphere stratifications and the Gauss map, Proceedings of the Royal Soc. Edinburgh, 95A (1983), 115-136.

[13] O. P. Shcherbak, Projectively dual space curves and Legendre singularities, Sel. Math. Sov 5 (1986), 391-421. 